ESAIM: M2AN 49 (2015) 953-976

DOI: $10.1051 / \mathrm{m} 2 \mathrm{an} / 2014059$
ESAIM: Mathematical Modelling and Numerical Analysis

www.esaim-m2an.org

\title{
CONVERGENCE OF A HIGH ORDER METHOD IN TIME AND SPACE FOR THE MISCIBLE DISPLACEMENT EQUATIONS*,**
}

\author{
Jizhou Li ${ }^{1}$, Beatrice Riviere ${ }^{1}$ and Noel Walkington ${ }^{2}$
}

\begin{abstract}
A numerical method is formulated and analyzed for solving the miscible displacement problem under low regularity assumptions. The scheme employs discontinuous Galerkin time stepping with mixed and interior penalty discontinuous Galerkin finite elements in space. The numerical approximations of the pressure, velocity, and concentration converge to the weak solution as the mesh size and time step tend to zero. To pass to the limit a compactness theorem is developed which generalizes the Aubin-Lions theorem to accommodate discontinuous functions both in space and in time.
\end{abstract}

Mathematics Subject Classification. 65M12,65M60.

Received October 31, 2013. Revised October 30, 2014.

Published online June 19, 2015.

\section{INTRODUCTION}

The miscible displacement problem arises in many applications, such as contamination of groundwater, or production of trapped oil in reservoirs by enhanced oil recovery. A solvent fluid is injected into a porous medium; it mixes with a resident fluid. The fluid mixture moves in the porous medium as a single phase flow, with a velocity that follows Darcy's law. The solvent concentration satisfies a convection-dominated parabolic problem, with a diffusion-dispersion matrix that depends on the fluid velocity in a non-linear fashion. The analysis of the miscible displacement problem is complicated by the fact that the diffusion-dispersion matrix is not uniformly bounded above.

This paper has two main contributions. We introduce and prove a general Aubin-Lions theorem valid for broken Sobolev spaces. Then, we obtain convergence of a high order method in time and in space for the solution of the miscible displacement problem under low regularity. The pressure and velocity equations are discretized by the mixed finite element method whereas the concentration equation is discretized by the interior penalty discontinuous Galerkin (IPDG) method. The time-stepping technique is the high order discontinuous Galerkin method.

\footnotetext{
Keywords and phrases. Generalized Aubin-Lions, discontinuous Galerkin, mixed finite element, arbitrary order, weak solution, convergence.

* This work was supported in part by the grant NSF-DMS 1318348.

** This work was supported by the NSF through the Center for Nonlinear Analysis.

1 Department of Computational and Applied Mathematics, Rice University, Houston, TX 77005, USA. Jizhou.Li@rice.edu; riviere@rice.edu

2 Department of Mathematics, Carnegie Mellon University, Pittsburgh, PA 15213, USA. noelw@andrew.cmu.edu
} 
There is little published work on the convergence analysis of numerical methods for solving the miscible displacement for non-smooth solutions and for the case of unbounded diffusion-dispersion matrix. In [17], we analyzed a method that employs the continuous finite element (FEM) method for the concentration equation and the mixed finite element (MFE) method for the pressure and velocity equations. In this present work, the discontinuous Galerkin (DG) method is used for approximating the concentration equation. It is well-known that the DG method is well-suited for convection dominated problems whereas the classical FEM yields spurious oscillations. To our knowledge, our work is the only one that analyzes an arbitrarily higher order in time method with DG in space for non-smooth solutions and unbounded diffusion-dispersion matrix. In [1], a first order Euler method in time is combined with MFE and a symmetric DG in space and the convergence analysis is obtained by applying the standard Aubin-Lions lemma to carefully constructed interpolated functional spaces. In the scheme in [1], the diffusion-dispersion matrix is projected onto the space of piecewise polynomial matrices. In addition, the penalty parameter depends on the shape regularity of the mesh and polynomial degree of the approximation space. The work of [1] was extended to a Crank-Nicolson time discretization in [15].

For smooth solutions and bounded diffusion-dispersion matrix, several methods have been formulated and analyzed for the miscible displacement: FEM [12,13,18], MFE [9], DG [10], finite volume [16] for instance. In [20], the proposed method enforces boundedness of the diffusion-dispersion matrix by using a cut-off operator.

\subsection{Overview and notation}

The next section introduces the miscible displacement problem, the assumptions on the data and the numerical scheme. Convergence of the numerical approximations is established in Section 3. A generalization of the Aubin-Lions theorem used in the convergence proof is proved in Section 4. Numerical examples are given in Section 5 .

Standard notation is used for the Lebesgue spaces, $L^{p}(\Omega)$, and Sobolev spaces, $W^{m, p}(\Omega), H^{1}(\Omega)=W^{1,2}(\Omega)$. The $L^{2}$ inner-product over a domain $\mathcal{O}$ is denoted by $(\cdot, \cdot)_{\mathcal{O}}$ and the subscript is dropped if $\mathcal{O}=\Omega$. The pressure will be in $L_{0}^{2}(\Omega)=\left\{q \in L^{2}(\Omega): \int_{\Omega} q=0\right\}$, and the velocity will belong to $H(\Omega$, div $)=\left\{\mathbf{v} \in L^{2}(\Omega)^{d}\right.$ : $\left.\operatorname{div}(\mathbf{v}) \in L^{2}(\Omega)\right\}$. The subspace of $H(\Omega ; \operatorname{div})$ with vanishing normal component on the boundary is denoted by $H_{0}(\Omega, \operatorname{div})=\{\mathbf{v} \in H(\Omega, \operatorname{div}): \mathbf{v} \cdot \mathbf{n}=0$ on $\partial \Omega\}$. The space of functions with bounded variation is denoted by

$$
B V(\Omega)=\left\{w \in L^{1}(\Omega) \mid \int_{\Omega} w \operatorname{div}(\phi) \leq C(w)\|\phi\|_{C(\bar{\Omega})}, \phi \in C_{c}^{1}(\bar{\Omega})^{d}\right\} .
$$

Solutions of evolution problems will be functions from $[0, T]$ into these spaces and the usual notation, $L^{2}\left[0, T ; H^{1}(\Omega)\right], C\left[0, T ; L^{2}(\Omega)\right]$, is used to indicate the temporal regularity of such functions. The numerical solutions will be constructed using polynomials; if $E \subset \Omega$ then $\mathcal{P}_{k}(E)$ denotes the set of all polynomials of degree less or equal to $k$ over $E$. Similarly, if $0 \leq a<b \leq T$ and $H$ is a function space then

$$
\mathcal{P}_{\ell}[a, b ; H]=\left\{\sum_{i=0}^{\ell} t^{i} v_{i} \mid t \in[a, b], v_{i} \in H, i=0, \ldots, \ell\right\} .
$$

Notation denoting jumps, averages, and specific finite element spaces used for the numerical scheme is introduced in Section 2.2. We write $a \lesssim b$ when there is a constant positive $M$ independent of the mesh parameters used for the numerical scheme such that $a \leq M b$.

\section{Problem AND SCHEME}

\subsection{Miscible displacement equations}

Let $[0, T]$ be a time interval and $\Omega \subset \Re^{d}$ be the region occupied by the porous medium in which a polymer solvent is being displaced. Under the assumption of incompressibility, the fluid pressure $p$ and velocity $\mathbf{u}$ satisfy 
the following equations

$$
\begin{array}{r}
\nabla \cdot \mathbf{u}=q^{I}-q^{P}, \quad \text { in } \quad \Omega \times(0, T), \\
\mathbf{u}=-\mathbf{K}(c)(\nabla p-\rho(c) \mathbf{g}), \quad \text { in } \quad \Omega \times(0, T) .
\end{array}
$$

The concentration $c$ of the solvent satisfies

$$
\partial_{t}(\phi c)-\operatorname{div}(\mathbf{D}(\mathbf{u}) \nabla c-c \mathbf{u})=q^{I} \hat{c}-q^{P} c, \quad \text { in } \quad \Omega \times(0, T) .
$$

The coefficients in the model are the injection $q^{I}$ and production $q^{P}$ functions, the fluid density $\rho(c)$, the gravity vector $\mathbf{g}$, the porosity of the medium $\phi$, the diffusion-dispersion matrix $\mathbf{D}(\mathbf{u})$, the injected concentration $\hat{c}$, and the matrix $\mathbf{K}(c)$, which is the ratio between the permeability matrix $\mathbf{k}$ and the fluid viscosity $\mu(c)$.

The numerical scheme introduced below uses the following reformulation of the diffusion equation for the concentration

$$
\partial_{t}(\phi c)-\operatorname{div}(\mathbf{D}(\mathbf{u}) \nabla c-(1 / 2) c \mathbf{u})+(1 / 2) \mathbf{u} \cdot \nabla c+(1 / 2)\left(q^{I}+q^{P}\right) c=q^{I} \hat{c} .
$$

The natural weak statement of this equation preserves the skew symmetric property of the operator $\operatorname{div}(c \mathbf{u})$ independently of equation (2.1). Equations (2.1) - (2.3) are then completed by boundary conditions

$$
\mathbf{u} \cdot \mathbf{n}=0, \quad \text { and } \quad \mathbf{D}(\mathbf{u}) \nabla c \cdot \mathbf{n}=0, \quad \text { on } \quad \partial \Omega \times(0, T),
$$

and the initial condition

$$
c(x, 0)=c_{0}(x), \quad x \in \Omega .
$$

Solutions of the numerical scheme will satisfy the following weak statement of equations $(2.1)-(2.3):(\mathbf{u}, p, c) \in$ $L^{\infty}\left[0, T ; H_{0}(\Omega, \operatorname{div})\right] \times L^{\infty}\left[0, T ; L_{0}^{2}(\Omega)\right] \times L^{2}\left[0, T ; H^{1}(\Omega)\right]$ and

$$
\begin{gathered}
\int_{0}^{T}\left(\mathbf{K}^{-1}(c) \mathbf{u}, \mathbf{v}\right)-(p, \operatorname{div}(\mathbf{v}))=\int_{0}^{T}(\rho(c) \mathbf{g}, \mathbf{v}), \\
\int_{0}^{T}(q, \operatorname{div}(\mathbf{u}))=\int_{0}^{T}\left(q^{I}-q^{P}, q\right), \\
\int_{0}^{T}\left(-\left(\phi c, \partial_{t} w\right)+(\mathbf{D}(\mathbf{u}) \nabla c-(1 / 2) c \mathbf{u}, \nabla w)\right. \\
\left.+(1 / 2)(\mathbf{u} \cdot \nabla c, w)+(1 / 2)\left(\left(q^{I}+q^{P}\right) c, w\right)\right)=\left(\phi c_{0}, w(0)\right)+\int_{0}^{T}\left(q^{I} \hat{c}, w\right),
\end{gathered}
$$

for all $(\mathbf{v}, q) \in L^{1}[0, T ; H(\Omega, \operatorname{div})] \times L^{1}\left[0, T ; L_{0}^{2}(\Omega)\right]$ and for all

$$
w \in\left\{w \in L^{4}\left[0, T ; W^{1,4}(\Omega)\right] \cap H^{1}\left[0, T ; H^{1}(\Omega)^{\prime}\right]: w(T)=0\right\} .
$$

The condition $w \in L^{4}\left[0, T ; W^{1,4}(\Omega)\right]$ on the test function is technical and is needed since $\mathbf{D}(\mathbf{u})$ is not bounded. Existence of weak solutions, with $\mathbf{D}(\mathbf{u})^{1 / 2} \nabla c \in L^{2}\left[0, T ; L^{2}(\Omega)\right]$ is established in $[7,14]$ under the following assumptions on the coefficients and data which guarantee that the weak statement is well defined.

\section{Assumption 2.1.}

(1) $\Omega \subset \Re^{d}$ is a bounded Lipschitz domain, $d=2$ or 3 .

(2) $\mathbf{K}: \Omega \times \Re \rightarrow \Re^{d \times d}$ is symmetric, Carathéodory (measurable in first argument and continuous in the second almost everywhere), uniformly bounded and elliptic. That is, there exist constants $0<k_{0}<k_{1}$ such that

$$
k_{0}|\xi|^{2} \leq \xi^{T} \mathbf{K}(x, c) \xi \leq k_{1}|\xi|^{2}, \quad \xi \in \Re^{d},(x, c) \in \Omega \times \Re,
$$

where $|\xi|$ denotes the Euclidean norm. The spatial dependence will be omitted below; $\mathbf{K}(c) \equiv \mathbf{K}(x, c)$. 
(3) $\mathbf{D}: \Omega \times \Re^{d} \rightarrow \Re^{d \times d}$ is Carathéodory, symmetric valued, and Lipschitz continuous in the second variable, and there exist constants $0<d_{0}<d_{1}$ such that

$$
d_{0}(1+|\mathbf{u}|)|\xi|^{2} \leq \xi^{T} \mathbf{D}(x, \mathbf{u}) \xi \leq d_{1}(1+|\mathbf{u}|)|\xi|^{2}, \quad(x, \mathbf{u}) \in \Omega \times \Re^{d}, \xi \in \Re^{d} .
$$

The spatial dependence will be omitted below; $\mathbf{D}(\mathbf{u}) \equiv \mathbf{D}(x, \mathbf{u})$.

(4) $\hat{c} \in L^{\infty}(\Omega), \phi \in L^{\infty}(\Omega)$ and $\phi_{0}<\phi<\phi_{1}$ for some positive constants $\phi_{0}, \phi_{1}$.

(5) $q^{I}, q^{P} \in L^{\infty}\left[0, T ; L^{2}(\Omega)\right]$ with $q^{I}, q^{P} \geq 0$ and $\int_{\Omega} q^{I}(x, t)=\int_{\Omega} q^{P}(x, t)$ for $t \in[0, T]$.

(6) There exist positive constants $\rho_{0}, \rho_{1}$ such that the function $\rho: \Re \rightarrow \Re$ is Lipschitz continuous and $\rho_{0} \leq \rho \leq \rho_{1}$.

\subsection{Numerical scheme}

Spatial approximations of (2.1) - (2.2) are constucted using the mixed finite element method and spatial approximations of equation (2.3) are developed using the interior penalty discontinuous Galerkin (IPDG) method. Discontinuous Galerkin time stepping will be used to discretize time [11].

Let $\left\{\mathcal{E}_{h}\right\}_{h>0}$ be a regular family of meshes of $\Omega$, where $h$ is maximum element diameter; the finite element subspaces are:

$$
\begin{aligned}
& \mathbf{U}_{h}=\left\{\mathbf{u}_{h} \in H_{0}(\Omega ; \operatorname{div})\left|\mathbf{u}_{h}\right|_{E} \in\left(\mathcal{P}_{k}(E)\right)^{d}+x \mathcal{P}_{k}(E), E \in \mathcal{E}_{h}\right\} \\
& P_{h}=\left\{q_{h} \in L^{2}(\Omega):\left.q_{h}\right|_{E} \in \mathcal{P}_{k}(E), E \in \mathcal{E}_{h}\right\} \\
& C_{h}=\left\{c_{h} \in L^{2}(\Omega):\left.c_{h}\right|_{E} \in \mathcal{P}_{r}(E), E \in \mathcal{E}_{h}\right\}
\end{aligned}
$$

For definiteness Raviart-Thomas spaces are used for the velocity; however, any classical mixed finite element spaces suffice; for example $B D M_{k}\left(\mathcal{E}_{h}\right)$ and $B D F M_{k}\left(\mathcal{E}_{h}\right)[3]$.

Let $\left\{t^{n}\right\}_{n=0}^{N} \simeq\left\{t_{h}^{n}\right\}_{n=0}^{N_{h}}$ be a family of partitions of $[0, T]$ that are quasi-uniform; i.e., there exists $\nu \in(0,1]$ such that

$$
\nu \Delta t \leq \min _{1 \leq n \leq N}\left(t^{n}-t^{n-1}\right), \quad \text { where } \quad \Delta t=\max _{1 \leq n \leq N}\left(t^{n}-t^{n-1}\right) .
$$

The numerical solutions are discontinuous in time and the jump of a function $v$ at time $t^{n}$ is denoted by $\left[v^{n}\right]_{t}$ :

$$
v_{+}^{n}=\lim _{\epsilon \downarrow 0} v\left(\cdot, t_{n}+\epsilon\right), v_{-}^{n}=\lim _{\epsilon \downarrow 0} v\left(\cdot, t_{n}-\epsilon\right),\left[v^{n}\right]_{t}=v_{+}^{n}-v_{-}^{n} .
$$

The numerical solution of the concentration is discontinuous across mesh elements. To define the jump [.] and average $\{\cdot\}$ of a discontinuous function let $\Gamma_{h}$ denote the set of interior faces. Then for each $e \in \Gamma_{h}$ fix a normal vector $\mathbf{n}_{e}$ and let $E_{+}^{e}$ and $E_{-}^{e}$ denote the neighboring elements such that $\mathbf{n}_{e}$ points from $E_{+}^{e}$ to $E_{-}^{e}$. Then

$$
\{v\}=\frac{\left.v\right|_{E_{+}^{e}}+\left.v\right|_{E_{-}^{e}}}{2}, \quad \text { and } \quad[v]=\left.v\right|_{E_{+}^{e}}-\left.v\right|_{E_{-}^{e}} .
$$

The broken Sobolev spaces are denoted by $W^{s, p}\left(\mathcal{E}_{h}\right)$ and let $H^{s}\left(\mathcal{E}_{h}\right)=W^{s, 2}\left(\mathcal{E}_{h}\right)$.

The norms on $H^{1}\left(\mathcal{E}_{h}\right)$ and $W^{1,4}\left(\mathcal{E}_{h}\right)$ are defined as

$$
\begin{aligned}
& \|v\|_{H^{1}\left(\mathcal{E}_{h}\right)}=\left(\|v\|_{L^{2}(\Omega)}^{2}+\sum_{E \in \mathcal{E}_{h}}\|\nabla v\|_{L^{2}(E)}^{2}+\sum_{e \in \Gamma_{h}} h^{-1}\|[v]\|_{L^{2}(e)}^{2}\right)^{1 / 2}, \\
& \|v\|_{W^{1,4}\left(\mathcal{E}_{h}\right)}=\left(\|v\|_{L^{4}(\Omega)}^{4}+\sum_{E \in \mathcal{E}_{h}}\|\nabla v\|_{L^{4}(E)}^{4}+\sum_{e \in \Gamma_{h}} h^{-3}\|[v]\|_{L^{4}(e)}^{4}\right)^{1 / 4} .
\end{aligned}
$$


The $L^{2}$ inner-product on $\mathcal{E}_{h}$ and $\Gamma_{h}$ are:

$$
(\cdot, \cdot)_{\mathcal{E}_{h}}=\sum_{E \in \mathcal{E}_{h}}(\cdot, \cdot)_{E}, \quad(\cdot, \cdot)_{\Gamma_{h}}=\sum_{e \in \Gamma_{h}}(\cdot, \cdot)_{e} .
$$

With this notation the numerical scheme becomes:

find $\mathbf{u}_{h} \in \mathcal{P}_{\ell}\left[t^{n-1}, t^{n} ; \mathbf{U}_{h}\right], p_{h} \in \mathcal{P}_{\ell}\left[t^{n-1}, t^{n} ; P_{h}\right], c_{h} \in P_{\ell}\left[t^{n-1}, t^{n} ; C_{h}\right]$, satisfying

$$
\begin{gathered}
\int_{t_{n-1}}^{t_{n}}\left(\left(\mathbf{K}^{-1}\left(c_{h}\right) \mathbf{u}_{h}, \mathbf{v}_{h}\right)-\left(p_{h}, \operatorname{div}\left(\mathbf{v}_{h}\right)\right)\right)=\int_{t_{n-1}}^{t_{n}}\left(\rho\left(c_{h}\right) \mathbf{g}, \mathbf{v}_{h}\right), \\
\int_{t_{n-1}}^{t_{n}}\left(q_{h}, \operatorname{div}\left(\mathbf{u}_{h}\right)\right)=\int_{t_{n-1}}^{t_{n}}\left(q^{I}-q^{P}, q_{h}\right), \\
\int_{t_{n-1}}^{t_{n}}\left(\left(\phi \partial_{t} c_{h}, w_{h}\right)+B_{d}\left(c_{h}, w_{h} ; \mathbf{u}_{h}\right)+B_{c q}\left(c_{h}, w_{h} ; \mathbf{u}_{h}\right)\right)+\left(\left[c_{h}^{n-1}\right]_{t}, \phi w_{h+1}^{n-1}\right)=\int_{t_{n-1}}^{t_{n}}\left(\hat{c} q^{I}, w_{h}\right),
\end{gathered}
$$

for all $\mathbf{v}_{h} \in \mathcal{P}_{\ell}\left[t^{n-1}, t^{n} ; \mathbf{U}_{h}\right], q_{h} \in \mathcal{P}_{\ell}\left[t^{n-1}, t^{n} ; P_{h}\right]$, and $w_{h} \in P_{\ell}\left[t^{n-1}, t^{n} ; C_{h}\right]$. Here $B_{d}(\cdot, \cdot ; \cdot)$ denotes the IPDG discretization of the operator $-\nabla \cdot(\mathbf{D}(\mathbf{u}) \nabla c)$ with penalty coefficient, $\sigma>0$, and parameter $\epsilon \in\{-1,0,1\}$,

$$
\begin{aligned}
B_{d}\left(c_{h}, w_{h} ; \mathbf{u}_{h}\right)=\left(\mathbf{D}\left(\mathbf{u}_{h}\right) \nabla c_{h}, \nabla w_{h}\right)_{\mathcal{E}_{h}}- & \left(\left[w_{h}\right],\left\{\mathbf{D}\left(\mathbf{u}_{h}\right) \nabla c_{h} \cdot \mathbf{n}_{e}\right\}\right)_{\Gamma_{h}} \\
& +\epsilon\left(\left[c_{h}\right],\left\{\mathbf{D}\left(\mathbf{u}_{h}\right) \nabla w_{h} \cdot \mathbf{n}_{e}\right\}\right)_{\Gamma_{h}}+\left(\sigma h^{-1}\left(1+\left\{\left|\mathbf{u}_{h}\right|\right\}\right)\left[c_{h}\right],\left[w_{h}\right]\right)_{\Gamma_{h}} .
\end{aligned}
$$

When the diffusion operator is unbounded, inclusion of the weight $1+\left\{\left|\mathbf{u}_{h}\right|\right\}$ in the last term is essential for our analysis. The term $B_{c q}(\cdot, \cdot ; \cdot)$ is the DG discretization of the convection terms,

$$
\begin{aligned}
B_{c q}\left(c_{h}, w_{h} ; \mathbf{u}_{h}\right)= & \frac{1}{2}\left(\left(\mathbf{u}_{h} \cdot \nabla c_{h}, w_{h}\right)_{\mathcal{E}_{h}}-\left(\mathbf{u}_{h} c_{h}, \nabla w_{h}\right)_{\mathcal{E}_{h}}+\left(\left(q^{I}+q^{P}\right) c_{h}, w_{h}\right)\right. \\
& \left.+\left(c_{h}^{\mathrm{up}} \mathbf{u}_{h} \cdot \mathbf{n}_{e},\left[w_{h}\right]\right)_{\Gamma_{h}}-\left(w_{h}^{\mathrm{down}} \mathbf{u}_{h} \cdot \mathbf{n}_{e},\left[c_{h}\right]\right)_{\Gamma_{h}}\right)
\end{aligned}
$$

The upwind value (resp. downwind value) of a discontinuous function $w_{h}$ with respect to $\mathbf{u}_{h} \cdot \mathbf{n}_{e}$ is denoted by $w_{h}^{\text {up }}$ (resp. $\left.w_{h}^{\text {down }}\right)$. To complete the definition of the scheme, set $c_{h-}^{0}$ to be the $L^{2}$ projection of the initial condition $c_{0}$. Our method is parametrized by $\epsilon$. As usual, the scheme is referred to as the SIPG method (NIPG method, IIPG method resp.) if $\epsilon=-1(\epsilon=+1, \epsilon=0$ resp.).

\section{Convergence of the scheme}

In this section, we establish the following theorem which is the main result of this paper.

Theorem 3.1. Let the data and coefficients satisfy Assumption 2.1, and let $\left\{\mathbf{u}_{h}, p_{h}, c_{h}\right\}_{h>0}$ be solutions of the discrete scheme (2.8)-(2.10) constructed over a regular family of meshes and quasi-uniform family of time partitions. If the maximal time step $\Delta t$ tends to zero with the mesh parameter $h$, then, upon passage to a subsequence, $\left\{\mathbf{u}_{h}, p_{h}, c_{h}\right\}_{h>0}$ converges strongly in $L^{2}[0, T ; H(\Omega ; \operatorname{div})] \times L^{2}\left[0, T ; L^{2}(\Omega)\right] \times L^{2}\left[0, T ; L^{2}(\Omega)\right]$, and the subsequence $\left\{\nabla c_{h}\right\}_{h>0}$ converges weakly in $L^{2}\left[0, T ; H^{-1}(\Omega)\right]$. In particular, the numerical solutions of SIPG discretization converge to a solution of the weak statement (2.4)-(2.6) of the miscible displacement equations.

The finite element spaces and time stepping scheme were chosen so that the discrete solutions inherit the stability properties of the continuous problem. The key step in the convergence proof is to then establish sufficient compactness to facilitate passage to the limit in the nonlinear terms. The following generalization of the Aubin-Lions theorem [19] is applicable in the current situation where:

(1) Solutions may be discontinuous in time; in particular, their time derivatives are not integrable. 
(2) Spatial operators are unbounded; that is, they are coercive over one space ( $V$ in the theorem), but continuous in a smaller space $(W \subset V)$.

(3) Discrete spaces are non-conforming ( $W_{h} \not \subset W$ in the theorem).

Theorem 3.2. Let $H$ be a Hilbert space with inner-product $(\cdot, \cdot)_{H}$ and $V$ and $W$ be Banach spaces equipped with norms $\|\cdot\|_{V}$ and $\|\cdot\|_{W}$. Assume that $W \subset H$ is dense and

$$
W \hookrightarrow V \hookrightarrow H \hookrightarrow W^{\prime}
$$

are dense embeddings with $V$ compactly embedded in $H$. The space $W^{\prime}$ denotes the dual space of $W$. Let $h \in$ $(0, \infty)$ be a (mesh) parameter and for each $h>0$ let $W\left(\mathcal{E}_{h}\right)$ be a Banach space with $W \hookrightarrow W\left(\mathcal{E}_{h}\right) \hookrightarrow V$ where the embedding constants are independent of $h$.

For each $h$, let $W_{h} \subset W\left(\mathcal{E}_{h}\right)$ be a closed subspace and let $\left\{t_{h}^{n}\right\}_{n=0}^{N_{h}}$ be a quasi-uniform family of partitions of $[0, T]$. Let $\Pi_{h}: H \rightarrow W_{h}$ denote the orthogonal projection, and assume that its restriction to $W\left(\mathcal{E}_{h}\right)$ is stable in the sense that there exists a constant $M>0$ independent of $h$ such that $\left\|\Pi_{h} w\right\|_{W\left(\mathcal{E}_{h}\right)} \leq M\|w\|_{W\left(\mathcal{E}_{h}\right)}$ for $w \in W\left(\mathcal{E}_{h}\right)$.

Fix $\ell \geq 0$ an integer and $1<p<\infty, 1 \leq q<\infty$, with $1 / p+1 / q \geq 1$, and assume that

(1) For each $h>0, w_{h} \in\left\{w_{h} \in L^{p}\left[0, T ; W_{h}\right]\left|w_{h}\right|_{\left(t_{h}^{n-1}, t^{n}\right)_{h}} \in \mathcal{P}_{\ell}\left[t_{h}^{n-1}, t_{h}^{n} ; W_{h}\right]\right\}$ and on each interval satisfies

$$
\forall z_{h} \in \mathcal{P}_{\ell}\left[t_{h}^{n-1}, t_{h}^{n} ; W_{h}\right], \quad \int_{t_{h}^{n-1}}^{t_{h}^{n}}\left(\partial_{t} w_{h}, z_{h}\right)_{H}+\left(w_{h+}^{n-1}-w_{h-}^{n-1}, z_{h+}^{n-1}\right)_{H}=\int_{t_{h}^{n-1}}^{t_{h}^{n}} F_{h}\left(z_{h}\right) .
$$

(2) The sequence $\left\{w_{h}\right\}_{h>0}$ is bounded in $L^{p}[0, T ; V]$.

(3) For each $h>0, F_{h} \in L^{q}\left[0, T ; W_{h}^{\prime}\right]$ and $\left\{\left\|F_{h}\right\|_{L^{q}\left[0, T ; W_{h}^{\prime}\right]}\right\}_{h>0} \subset \Re$ is bounded.

Then the set $\left\{w_{h}\right\}_{h>0}$ is precompact in $L^{p}[0, T ; H] \cap L^{r}\left[0, T ; W^{\prime}\right]$ for each $1 \leq r<\infty$.

This theorem is proved in Section 4 and extends of the corresponding results in [17,21] for conforming approximations, $W_{h} \subset W$, to the non-conforming setting, $W_{h} \not \subset W$. In this situation it is necessary introduce the semi-discrete space $W\left(\mathcal{E}_{h}\right)$ which contains both $W$ and $W_{h}$. Solutions of the time stepping scheme are equicontinuous in the (mesh dependent) spaces $L^{r}\left[0, T ; W_{h}^{\prime}\right]$, and the structural assumptions relating the three spaces, $W_{h}, W\left(\mathcal{E}_{h}\right)$, and $H$, are used to establish equicontinuity in $L^{r}\left[0, T ; W^{\prime}\right]$. Compactness then follows from standard arguments.

\subsection{Stability of the numerical solutions}

Stability estimates for the Darcy velocity and pressure computed using the mixed finite element method are well-known [4] The following lemma from [17] bounds the numerical approximations of the velocity and pressure by the data.

Lemma 3.3 (Stability of the pressure and the velocity). Let the coefficients and data satisfy Assumption 2.1. Then exists a constant $M>0$ depending only upon the aspect ratio of the mesh $\mathcal{E}_{h}$ such that the velocity and pressure computed using equations (2.8) and (2.9) satisfy the following bounds.

- If $1 \leq p, q \leq \infty$ and $q^{I}, q^{P} \in L^{p}\left[0, T ; L^{q}(\Omega)\right]$, then

$$
\left\|\operatorname{div}\left(\mathbf{u}_{h}\right)\right\|_{L^{p}\left[0, T ; L^{q}(\Omega)\right]} \leq M\left(\left\|q^{I}\right\|_{L^{p}\left[0, T ; L^{q}(\Omega)\right]}+\left\|q^{P}\right\|_{L^{p}\left[0, T ; L^{q}(\Omega)\right]}\right) .
$$

- If $1 \leq p \leq \infty, q^{I}, q^{P} \in L^{p}\left[0, T ; L^{2}(\Omega)\right]$, then

$$
\left\|\mathbf{u}_{h}\right\|_{L^{p}[0, T ; H(\Omega, \mathrm{div})]}+\left\|p_{h}\right\|_{L^{p}\left[0, T ; L^{2}(\Omega)\right]} \leq M\left(\left\|q^{I}\right\|_{L^{p}\left[0, T ; L^{2}(\Omega)\right]}+\left\|q^{P}\right\|_{L^{p}\left[0, T ; L^{2}(\Omega)\right]}+\left\|\rho_{1} \mathbf{g}\right\|_{L^{p}\left[0, T ; L^{2}(\Omega)\right]}\right) .
$$


The following semi-norm on $C_{h}$ is used to characterize stability bound of the concentration.

$$
\left\|v_{h}\right\|_{C_{h}}^{2}=\sum_{E \in \mathcal{E}_{h}}\left\|\mathbf{D}^{1 / 2}\left(\mathbf{u}_{h}\right) \nabla v_{h}\right\|_{L^{2}(E)}^{2}+\sum_{e \in \Gamma_{h}} h^{-1}\left\|\left(1+\left\{\left|\mathbf{u}_{h}\right|\right\}\right)^{1 / 2}\left[v_{h}\right]\right\|_{L^{2}(e)}^{2}, \quad v_{h} \in C_{h} .
$$

The next two lemmas state the continuity and coercivity of the forms $B_{d}$ and $B_{c q}$. The proof of these lemmas is given at the end of the section.

Lemma 3.4 (Continuity properties). Let $c_{h}$ and $w_{h}$ be in $C_{h}$ and let $\mathbf{u}_{h}$ be in $\mathbf{U}_{h}$.

$$
\begin{aligned}
& \left|B_{d}\left(c_{h}, w_{h} ; \mathbf{u}_{h}\right)\right| \lesssim\left(1+\left\|\mathbf{u}_{h}\right\|_{L^{2}(\Omega)}^{1 / 2}\right)\left\|c_{h}\right\|_{C_{h}}\left\|w_{h}\right\|_{W^{1,4}\left(\mathcal{E}_{h}\right)}, \\
& \left|B_{c q}\left(c_{h}, w_{h} ; \mathbf{u}_{h}\right)\right| \lesssim\left\|w_{h}\right\|_{W^{1,4}\left(\mathcal{E}_{h}\right)}\left(\left\|\mathbf{u}_{h}\right\|_{L^{2}(\Omega)}^{1 / 2}\left\|c_{h}\right\|_{C_{h}}+\left(\left\|q^{I}+q^{P}\right\|_{L^{2}(\Omega)}+\left\|\mathbf{u}_{h}\right\|_{L^{2}(\Omega)}\right)\left\|c_{h}\right\|_{L^{4}(\Omega)}\right) .
\end{aligned}
$$

Lemma 3.5 (Coercivity properties). Let $c_{h}$ and $w_{h}$ be in $C_{h}$ and let $\mathbf{u}_{h}$ be in $\mathbf{U}_{h}$. Then there exists $\sigma_{0}>0$ independent of $h$ provided $\sigma \geq \sigma_{0}$ if the symmetrization parameter $\epsilon$ takes the value -1 or 0 , and $\sigma=1$ if $\epsilon$ is equal to 1 such that

$$
\begin{aligned}
& B_{d}\left(w_{h}, w_{h} ; \mathbf{u}_{h}\right) \geq \frac{1}{2}\left\|w_{h}\right\|_{C_{h}}^{2}, \\
& B_{c q}\left(w_{h}, w_{h} ; \mathbf{u}_{h}\right) \geq \frac{1}{2}\left(\left\|\left(q^{I}+q^{P}\right)^{1 / 2} w_{h}\right\|_{L^{2}(\Omega)}^{2}+\left\|\left|\mathbf{u}_{h} \cdot \mathbf{n}_{e}\right|^{1 / 2}\left[w_{h}\right]\right\|_{\Gamma_{h}}^{2}\right) .
\end{aligned}
$$

Theorem 3.6 (Stability of the concentration). There exists $\sigma_{0}>0$ independent of $h$ such that solutions of equation (2.10) satisfy

$$
\begin{aligned}
& \left\|\phi^{1 / 2} c_{h-}^{m}\right\|_{L^{2}(\Omega)}^{2}+\int_{0}^{t^{m}}\left(\left\|c_{h}\right\|_{C_{h}}^{2}+\left\|\sqrt{q^{P}} c_{h}\right\|_{L^{2}(\Omega)}^{2}+\left\|\left|\mathbf{u}_{h} \cdot \mathbf{n}_{e}\right|^{1 / 2}\left[c_{h}\right]\right\|_{\Gamma_{h}}^{2}\right) \\
& +\sum_{n=1}^{m}\left\|\left[\phi^{1 / 2} c_{h}^{n-1}\right]_{t}\right\|_{L^{2}(\Omega)}^{2} \leq\left\|\phi^{1 / 2} c_{h-}^{0}\right\|_{L^{2}(\Omega)}^{2}+\int_{0}^{t^{m}}\left\|\sqrt{q^{I}} \hat{c}\right\|_{L^{2}(\Omega)}^{2},
\end{aligned}
$$

provided $\sigma \geq \sigma_{0}$ if the symmetrization parameter $\epsilon$ takes the value -1 or 0 , and $\sigma=1$ if $\epsilon$ is equal to 1 . In particular, $\max _{1 \leq n \leq N}\left\|c_{h-}^{n}\right\|_{L^{2}(\Omega)},\left\|c_{h}\right\|_{L^{2}\left[0, T ; C_{h}\right]}$, and $\left\|c_{h}\right\|_{L^{2}\left[0, T ; H^{1}\left(\mathcal{E}_{h}\right)\right]}$ are bounded independently of $h$ and $\Delta t$.

Proof. We integrate the time-derivative to obtain:

$$
\begin{array}{r}
\int_{t_{n-1}}^{t_{n}}\left(\phi \partial_{t} c_{h}, c_{h}\right) \mathrm{d} t+\left(c_{h+}^{n-1}, \phi c_{h+}^{n-1}\right)=\frac{1}{2}\left(\phi c_{h-}^{n}, c_{h-}^{n}\right)+\frac{1}{2}\left(\phi c_{h+}^{n-1}, c_{h+}^{n-1}\right) \\
=\frac{1}{2}\left\|\phi^{1 / 2} c_{h-}^{n}\right\|_{L^{2}(\Omega)}^{2}+\frac{1}{2}\left\|\left[\phi^{1 / 2} c_{h}^{n-1}\right]_{t}\right\|_{L^{2}(\Omega)}^{2}+\left(\phi c_{h+}^{n-1}, c_{h-}^{n-1}\right)-\frac{1}{2}\left\|\phi^{1 / 2} c_{h-}^{n-1}\right\|_{L^{2}(\Omega)}^{2} .
\end{array}
$$

Therefore, taking $w_{h}=c_{h}$ in (2.10) and using (3.7), we obtain

$$
\begin{array}{r}
\int_{t_{n-1}}^{t_{n}}\left(B_{d}\left(c_{h}, c_{h} ; \mathbf{u}_{h}\right)+B_{c q}\left(c_{h}, c_{h} ; \mathbf{u}_{h}\right)\right)+\frac{1}{2}\left\|\phi^{1 / 2} c_{h-}^{n}\right\|_{L^{2}(\Omega)}^{2}+\frac{1}{2}\left\|\left[\phi^{1 / 2} c_{h}^{n-1}\right]_{t}\right\|_{L^{2}(\Omega)}^{2} \\
+\left(\phi c_{h+}^{n-1}, c_{h-}^{n-1}\right)-\frac{1}{2}\left\|\phi^{1 / 2} c_{h-}^{n-1}\right\|_{L^{2}(\Omega)}^{2}=\left(c_{h-}^{n-1}, \phi c_{h+}^{n-1}\right)+\int_{t_{n-1}}^{t_{n}}\left(\hat{c} q^{I}, c_{h}\right) .
\end{array}
$$

Hence, we obtain after simplification

$$
\begin{aligned}
\frac{1}{2}\left\|\phi^{1 / 2} c_{h-}^{n}\right\|_{L^{2}(\Omega)}^{2}+\frac{1}{2}\left\|\left[\phi^{1 / 2} c_{h}^{n-1}\right]_{t}\right\|_{L^{2}(\Omega)}^{2} & +\int_{t_{n-1}}^{t_{n}}\left(B_{d}\left(c_{h}, c_{h} ; \mathbf{u}_{h}\right)+B_{c q}\left(c_{h}, c_{h} ; \mathbf{u}_{h}\right)\right) \\
& =\frac{1}{2}\left\|\phi^{1 / 2} c_{h-}^{n-1}\right\|_{L^{2}(\Omega)}^{2}+\int_{t_{n-1}}^{t_{n}}\left(\hat{c} q^{I}, c_{h}\right) .
\end{aligned}
$$


Using (3.4) and (3.5), the equation above becomes an inequality

$$
\begin{array}{r}
\frac{1}{2}\left\|\phi^{1 / 2} c_{h-}^{n}\right\|_{L^{2}(\Omega)}^{2}+\frac{1}{2} \int_{t_{n-1}}^{t_{n}}\left(\left\|c_{h}\right\|_{C_{h}}^{2}+\left\|\left(q^{I}+q^{P}\right)^{1 / 2} c_{h}\right\|_{L^{2}(\Omega)}^{2}+\left\|\left.\mathbf{u}_{h} \cdot \mathbf{n}_{e}\right|^{1 / 2}\left[c_{h}\right]\right\|_{\Gamma_{h}}^{2}\right) \\
+\frac{1}{2}\left\|\left[\phi^{1 / 2} c_{h}^{n-1}\right]_{t}\right\|_{L^{2}(\Omega)}^{2} \leq \frac{1}{2}\left\|\phi^{1 / 2} c_{h-}^{n-1}\right\|_{L^{2}(\Omega)}^{2}+\int_{t_{n-1}}^{t_{n}}\left(\hat{c} q^{I}, c_{h}\right) .
\end{array}
$$

Now, again use Cauchy-Schwarz's inequality and Young's inequality to obtain

$$
\left(\hat{c} q^{I}, c_{h}\right) \leq \frac{1}{2}\left\|\sqrt{q^{I}} c_{h}\right\|_{L^{2}(\Omega)}^{2}+\frac{1}{2}\left\|\hat{c} \sqrt{q^{I}}\right\|_{L^{2}(\Omega)}^{2} .
$$

Thus, substitute this term into (3.8)

$$
\begin{gathered}
\left\|\phi^{1 / 2} c_{h-}^{n}\right\|_{L^{2}(\Omega)}^{2}+\left\|\left[\phi^{1 / 2} c_{h}^{n-1}\right]_{t}\right\|_{L^{2}(\Omega)}^{2}+\int_{t_{n-1}}^{t_{n}}\left(\left\|c_{h}\right\|_{C_{h}}^{2}+\left\|\sqrt{q^{P}} c_{h}\right\|_{L^{2}(\Omega)}^{2}+\left\|\left|\mathbf{u}_{h} \cdot \mathbf{n}_{e}\right|^{1 / 2}\left[c_{h}\right]\right\|_{\Gamma_{h}}^{2}\right) \\
\leq\left\|\phi^{1 / 2} c_{h-}^{n-1}\right\|_{L^{2}(\Omega)}^{2}+\int_{t_{n-1}}^{t_{n}}\left\|\sqrt{q^{I}} \hat{c}\right\|_{L^{2}(\Omega)}^{2} .
\end{gathered}
$$

We sum up over all time steps to obtain (3.6). Since $q^{P}$ is non-negative, the definition of $\|\cdot\|_{H^{1}\left(\mathcal{E}_{h}\right)}$ and $\|\cdot\|_{C_{h}}$, with (3.6) gives the uniform bound of $\left\|c_{h}\right\|_{L^{2}\left[0, T ; H^{1}\left(\mathcal{E}_{h}\right)\right]}$ independent of $h$ and $\Delta t$.

The proof of Lemma 3.4 is now given.

Proof. The first term of $B_{d}\left(c_{h}, w_{h} ; \mathbf{u}_{h}\right)$ is

$$
\left(\mathbf{D}\left(\mathbf{u}_{h}\right) \nabla c_{h}, \nabla w_{h}\right)_{\mathcal{E}_{h}} \leq \sum_{E \in \mathcal{E}_{h}}\left\|\mathbf{D}^{1 / 2}\left(\mathbf{u}_{h}\right) \nabla c_{h}\right\|_{L^{2}(E)}\left\|\mathbf{D}^{1 / 2}\left(\mathbf{u}_{h}\right) \nabla w_{h}\right\|_{L^{2}(E)} .
$$

Notice that by $(2.7)$,

$$
\begin{aligned}
\left\|\mathbf{D}^{1 / 2}\left(\mathbf{u}_{h}\right) \nabla w_{h}\right\|_{L^{2}(E)} & \lesssim\left(\int_{E}\left(1+\left|\mathbf{u}_{h}\right|\right)\left|\nabla w_{h}\right|^{2}\right)^{1 / 2} \lesssim\left\|\nabla w_{h}\right\|_{L^{2}(E)}+\left(\int_{E}\left|\mathbf{u}_{h}\right|\left|\nabla w_{h}\right|^{2}\right)^{1 / 2} \\
& \lesssim\left\|\nabla w_{h}\right\|_{L^{2}(E)}+\left\|\mathbf{u}_{h}\right\|_{L^{2}(E)}^{1 / 2}\left\|\nabla w_{h}\right\|_{L^{4}(E)} .
\end{aligned}
$$

So, we have

$$
\begin{gathered}
\left(\mathbf{D}\left(\mathbf{u}_{h}\right) \nabla c_{h}, \nabla w_{h}\right)_{\mathcal{E}_{h}} \lesssim \sum_{E \in \mathcal{E}_{h}}\left\|\mathbf{D}^{1 / 2}\left(\mathbf{u}_{h}\right) \nabla c_{h}\right\|_{L^{2}(E)}\left(\left\|\nabla w_{h}\right\|_{L^{2}(E)}+\left\|\mathbf{u}_{h}\right\|_{L^{2}(E)}^{1 / 2}\left\|\nabla w_{h}\right\|_{L^{4}(E)}\right) \\
\lesssim\left\|\mathbf{D}^{1 / 2}\left(\mathbf{u}_{h}\right) \nabla c_{h}\right\|_{L^{2}\left(\mathcal{E}_{h}\right)}\left(\left\|\nabla w_{h}\right\|_{L^{2}\left(\mathcal{E}_{h}\right)}+\left(\sum_{E \in \mathcal{E}_{h}}\left\|\mathbf{u}_{h}\right\|_{L^{2}(E)}\left\|\nabla w_{h}\right\|_{L^{4}(E)}^{2}\right)^{1 / 2}\right) \\
\lesssim\left\|\mathbf{D}^{1 / 2}\left(\mathbf{u}_{h}\right) \nabla c_{h}\right\|_{L^{2}\left(\mathcal{E}_{h}\right)}\left(\left\|\nabla w_{h}\right\|_{L^{2}\left(\mathcal{E}_{h}\right)}+\left\|\mathbf{u}_{h}\right\|_{L^{2}(\Omega)}^{1 / 2}\left\|\nabla w_{h}\right\|_{L^{4}\left(\mathcal{E}_{h}\right)}\right) .
\end{gathered}
$$

And consequently using the fact that

$$
\left\|\nabla w_{h}\right\|_{L^{2}\left(\mathcal{E}_{h}\right)} \lesssim\left\|\nabla w_{h}\right\|_{L^{4}\left(\mathcal{E}_{h}\right)}
$$

we have,

$$
\left(\mathbf{D}\left(\mathbf{u}_{h}\right) \nabla c_{h}, \nabla w_{h}\right)_{\mathcal{E}_{h}} \lesssim\left\|c_{h}\right\|_{C_{h}}\left(1+\left\|\mathbf{u}_{h}\right\|_{L^{2}(\Omega)}^{1 / 2}\right)\left\|w_{h}\right\|_{W^{1,4}\left(\mathcal{E}_{h}\right)} .
$$


For the term $\left(\left[w_{h}\right],\left\{\mathbf{D}\left(\mathbf{u}_{h}\right) \nabla c_{h} \cdot \mathbf{n}_{e}\right\}\right)_{\Gamma_{h}}$ using the same argument as for (3.26) we have,

$$
\left(\left[w_{h}\right],\left\{\mathbf{D}\left(\mathbf{u}_{h}\right) \nabla c_{h} \cdot \mathbf{n}_{e}\right\}\right)_{\Gamma_{h}} \lesssim \sum_{e \in \Gamma_{h}}\left(\int_{e} h^{-1}\left(1+\left\{\left|\mathbf{u}_{h}\right|\right\}\right)\left[w_{h}\right]^{2}\right)^{1 / 2}\left\|\mathbf{D}^{1 / 2}\left(\mathbf{u}_{h}\right) \nabla c_{h}\right\|_{L^{2}\left(\mathcal{E}_{h}\right)} .
$$

We have the following inequality:

$$
\left(\sum_{e \in \Gamma_{h}} \int_{e} h^{-1}\left(1+\left\{\left|\mathbf{u}_{h}\right|\right\}\right)\left[w_{h}\right]^{2}\right)^{1 / 2} \lesssim\left(\sum_{e \in \Gamma_{h}} \int_{e} h^{-1}\left[w_{h}\right]^{2}\right)^{1 / 2}+\left(\sum_{e \in \Gamma_{h}} \int_{e} h^{-1}\left\{\left|\mathbf{u}_{h}\right|\right\}\left[w_{h}\right]^{2}\right)^{1 / 2} .
$$

Using Cauchy-Schwarz's inequality and the fact that $|e| \approx h^{d-1}$, we have

$$
\sum_{e \in \Gamma_{h}} \int_{e} h^{-1}\left[w_{h}\right]^{2} \lesssim\left(\sum_{e \in \Gamma_{h}} h^{-3} \int_{e}\left[w_{h}\right]^{4}\right)^{1 / 2}\left(\sum_{e \in \Gamma_{h}} h^{d}\right)^{1 / 2} \lesssim\left(\sum_{e \in \Gamma_{h}} h^{-3} \int_{e}\left[w_{h}\right]^{4}\right)^{1 / 2} .
$$

For the other term, we can write

$$
\sum_{e \in \Gamma_{h}} \int_{e} h^{-1}\left\{\left|\mathbf{u}_{h}\right|\right\}\left[w_{h}\right]^{2} \lesssim \sum_{e \in \Gamma_{h}} \int_{e} h^{-1}\left|\mathbf{u}_{h}^{+}\right|\left[w_{h}\right]^{2}+\sum_{e \in \Gamma_{h}} \int_{e} h^{-1}\left|\mathbf{u}_{h}^{-}\right|\left[w_{h}\right]^{2} .
$$

We treat each term separately, but in a similar fashion

$$
\begin{gathered}
\sum_{e \in \Gamma_{h}} \int_{e} h^{-1}\left|\mathbf{u}_{h}^{+}\right|\left[w_{h}\right]^{2} \lesssim\left(\sum_{e \in \Gamma_{h}} h^{-3} \int_{e}\left[w_{h}\right]^{4}\right)^{1 / 2}\left(\sum_{e \in \Gamma_{h}} h \int_{e}\left|\mathbf{u}_{h}^{+}\right|^{2}\right)^{1 / 2} \\
\lesssim\left(\sum_{e \in \Gamma_{h}} h^{-3} \int_{e}\left[w_{h}\right]^{4}\right)^{1 / 2}\left(\sum_{e \in \Gamma_{h}}\left\|\mathbf{u}_{h}\right\|_{L^{2}\left(E_{+}^{e}\right)}^{2}\right)^{1 / 2}
\end{gathered}
$$

Therefore we have

$$
\sum_{e \in \Gamma_{h}} \int_{e} h^{-1}\left\{\left|\mathbf{u}_{h}\right|\right\}\left[w_{h}\right]^{2} \lesssim\left\|\mathbf{u}_{h}\right\|_{L^{2}(\Omega)}\left(\sum_{e \in \Gamma_{h}} h^{-3} \int_{e}\left[w_{h}\right]^{4}\right)^{1 / 2}
$$

To summarize, from (3.12) and (3.13), we have,

$$
\left(\sum_{e \in \Gamma_{h}} h^{-1} \int_{e}\left(1+\left\{\left|\mathbf{u}_{h}\right|\right\}\right)\left[w_{h}\right]^{2}\right)^{1 / 2} \lesssim\left(1+\left\|\mathbf{u}_{h}\right\|_{L^{2}(\Omega)}^{1 / 2}\right)\left(\sum_{e \in \Gamma_{h}} h^{-3} \int_{e}\left[w_{h}\right]^{4}\right)^{1 / 4}
$$

and thus,

$$
\left(\left[w_{h}\right],\left\{\mathbf{D}\left(\mathbf{u}_{h}\right) \nabla c_{h} \cdot \mathbf{n}_{e}\right\}\right)_{\Gamma_{h}} \lesssim\left(1+\left\|\mathbf{u}_{h}\right\|_{L^{2}(\Omega)}^{1 / 2}\right)\left\|w_{h}\right\|_{W\left(\mathcal{E}_{h}\right)}\left\|\mathbf{D}^{1 / 2}\left(\mathbf{u}_{h}\right) \nabla c_{h}\right\|_{L^{2}\left(\mathcal{E}_{h}\right)} .
$$

For the third term of $B_{d}\left(c_{h}, w_{h} ; \mathbf{u}_{h}\right)$, we use a trace inequality (see (A.2) in Appendix) and a similar argument as in $(3.25)$

$$
\begin{gathered}
\left(\left[c_{h}\right],\left\{\mathbf{D}\left(\mathbf{u}_{h}\right) \nabla w_{h} \cdot \mathbf{n}_{e}\right\}\right)_{\Gamma_{h}}=\sum_{e \in \Gamma_{h}}\left(\left[c_{h}\right],\left\{\mathbf{D}\left(\mathbf{u}_{h}\right) \nabla w_{h} \cdot \mathbf{n}_{e}\right\}\right)_{e} \\
\lesssim \sum_{e \in \Gamma_{h}}\left(\int_{e} h^{-1}\left(1+\left\{\left|\mathbf{u}_{h}\right|\right\}\right)\left[c_{h}\right]^{2}\right)^{1 / 2}\left\{\left\|\nabla w_{h}\right\|_{L^{2}\left(E^{e}\right)}+\left\|\mathbf{u}_{h}\right\|_{L^{2}\left(E^{e}\right)}^{1 / 2}\left\|\nabla w_{h}\right\|_{L^{4}\left(E^{e}\right)}\right\} \\
\lesssim \sum_{e \in \Gamma_{h}}\left(\int_{e} h^{-1}\left(1+\left\{\left|\mathbf{u}_{h}\right|\right\}\right)\left[c_{h}\right]^{2}\right)^{1 / 2}\left(\left\{\left\|\nabla w_{h}\right\|_{L^{2}\left(E^{e}\right)}\right\}+\left\{\left\|\mathbf{u}_{h}\right\|_{L^{2}\left(E^{e}\right)}^{1 / 2}\left\|\nabla w_{h}\right\|_{L^{4}\left(E^{e}\right)}\right\}\right) \\
\lesssim\left\|c_{h}\right\|_{C_{h}}\left(\left\|\nabla w_{h}\right\|_{L^{2}\left(\mathcal{E}_{h}\right)}+\left\|\mathbf{u}_{h}\right\|_{L^{2}(\Omega)}^{1 / 2}\left\|\nabla w_{h}\right\|_{L^{4}\left(\mathcal{E}_{h}\right)}\right) .
\end{gathered}
$$


Using Cauchy-Schwarz's inequality and (3.14), the penalty term in $B_{d}\left(c_{h}, w_{h} ; \mathbf{u}_{h}\right)$ can be bounded as

$$
\begin{aligned}
\left(\sigma h^{-1}\left(1+\left\{\left|\mathbf{u}_{h}\right|\right\}\right)\left[c_{h}\right],\left[w_{h}\right]\right)_{\Gamma_{h}} \lesssim & \left(\sum_{e \in \Gamma_{h}} \int_{e} h^{-1}\left(1+\left\{\left|\mathbf{u}_{h}\right|\right\}\right)\left[c_{h}\right]^{2}\right)^{1 / 2}\left(\sum_{e \in \Gamma_{h}} \int_{e} h^{-1}\left(1+\left\{\left|\mathbf{u}_{h}\right|\right\}\right)\left[w_{h}\right]^{2}\right)^{1 / 2} \\
& \lesssim\left\|c_{h}\right\|_{C_{h}}\left(1+\left\|\mathbf{u}_{h}\right\|_{L^{2}(\Omega)}^{1 / 2}\right)\left\|w_{h}\right\|_{W\left(\mathcal{E}_{h}\right)}
\end{aligned}
$$

Therefore the bound (3.2) is obtained by combining (3.9), (3.10), (3.15), (3.16) and (3.17).

To obtain (3.3), we now bound each term in $B_{c q}\left(c_{h}, w_{h} ; \mathbf{u}_{h}\right)$. For the first term, using (2.7), we have:

$$
\begin{aligned}
\left(\mathbf{u}_{h} \nabla c_{h}, w_{h}\right)_{\mathcal{E}_{h}} & \leq \sum_{E \in \mathcal{E}_{h}}\left(\int_{E}\left|\mathbf{u}_{h}\right|\left|\nabla c_{h}\right|^{2}\right)^{1 / 2}\left(\int_{E}\left|\mathbf{u}_{h}\right| w_{h}^{2}\right)^{1 / 2} \\
& \lesssim \sum_{E \in \mathcal{E}_{h}}\left\|\mathbf{D}^{1 / 2}\left(\mathbf{u}_{h}\right) \nabla c_{h}\right\|_{L^{2}(E)}\left\|\mathbf{u}_{h}\right\|_{L^{2}(E)}^{1 / 2}\left\|w_{h}\right\|_{L^{4}(E)} \\
& \lesssim\left\|\mathbf{D}^{1 / 2}\left(\mathbf{u}_{h}\right) \nabla c_{h}\right\|_{L^{2}\left(\mathcal{E}_{h}\right)}\left\|\mathbf{u}_{h}\right\|_{L^{2}(\Omega)}^{1 / 2}\left\|w_{h}\right\|_{L^{4}(\Omega)}
\end{aligned}
$$

Similarly we have

$$
\begin{aligned}
\left(\mathbf{u}_{h} c_{h}, \nabla w_{h}\right)_{\mathcal{E}_{h}} & \leq \sum_{E \in \mathcal{E}_{h}}\left\|\mathbf{u}_{h}\right\|_{L^{2}(E)}\left\|c_{h}\right\|_{L^{4}(E)}\left\|\nabla w_{h}\right\|_{L^{4}(E)} \\
& \leq\left\|\nabla w_{h}\right\|_{L^{4}\left(\mathcal{E}_{h}\right)}\left\|\mathbf{u}_{h}\right\|_{L^{2}(\Omega)}\left\|c_{h}\right\|_{L^{4}(\Omega)}
\end{aligned}
$$

For the third term in $B_{c q}\left(c_{h}, w_{h} ; \mathbf{u}_{h}\right)$ we easily obtain

$$
\left(\left(q^{I}+q^{P}\right) c_{h}, w_{h}\right) \leq\left\|q^{I}+q^{P}\right\|_{L^{2}(\Omega)}\left\|c_{h}\right\|_{L^{4}(\Omega)}\left\|w_{h}\right\|_{L^{4}(\Omega)} .
$$

For the upwind term, we remark that

$$
\left|c_{h}^{\mathrm{up}}\right| \leq \max \left\{\left|c_{h}^{+}\right|,\left|c_{h}^{-}\right|\right\} \leq\left|c_{h}^{+}\right|+\left|c_{h}^{-}\right| .
$$

Therefore, using the fact that $\mathbf{u}_{h}^{+} \cdot \mathbf{n}_{e}=\mathbf{u}_{h}^{-} \cdot \mathbf{n}_{e}$, we can write

$$
\left(c_{h}^{\mathrm{up}} \mathbf{u}_{h} \cdot \mathbf{n}_{e},\left[w_{h}\right]\right)_{e} \leq \int_{e}\left|c_{h}^{+}\right|\left|\mathbf{u}_{h}^{+}\right|\left|\left[w_{h}\right]\right|+\int_{e}\left|c_{h}^{-}\right|\left|\mathbf{u}_{h}^{-}\right|\left|\left[w_{h}\right]\right| .
$$

We treat each term separately but in a similar fashion. By Cauchy-Schwarz's inequality and trace inequalities, we have

$$
\begin{aligned}
\int_{e}\left|c_{h}^{+}\right|\left|\mathbf{u}_{h}^{+}\right|\left|\left[w_{h}\right]\right| & \leq\left(\int_{e}\left|\mathbf{u}_{h}^{+}\right|\left|c_{h}^{+}\right|^{2}\right)^{1 / 2}\left(\int_{e}\left|\mathbf{u}_{h}^{+}\right|\left[w_{h}\right]^{2}\right)^{1 / 2} \\
& \lesssim\left\|\mathbf{u}_{h}\right\|_{L^{2}\left(E_{+}^{e}\right)}^{1 / 2}\left\|c_{h}\right\|_{L^{4}\left(E_{+}^{e}\right)}\left(h^{-1} \int_{e}\left\{\left|\mathbf{u}_{h}\right|\right\}\left[w_{h}\right]^{2}\right)^{1 / 2} .
\end{aligned}
$$

Next, we sum up over all interior faces and obtain

$$
\sum_{e \in \Gamma_{h}}\left(c_{h}^{\mathrm{up}} \mathbf{u}_{h} \cdot \mathbf{n}_{e},\left[w_{h}\right]\right)_{e} \lesssim\left\|\mathbf{u}_{h}\right\|_{L^{2}(\Omega)}^{1 / 2}\left\|c_{h}\right\|_{L^{4}(\Omega)}\left(\sum_{e \in \Gamma_{h}} h^{-1} \int_{e}\left\{\left|\mathbf{u}_{h}\right|\right\}\left[w_{h}\right]^{2}\right)^{1 / 2},
$$


which, with (3.13), yields

$$
\left(c_{h}^{\mathrm{up}} \mathbf{u}_{h} \cdot \mathbf{n}_{e},\left[w_{h}\right]\right)_{\Gamma_{h}} \lesssim\left\|\mathbf{u}_{h}\right\|_{L^{2}(\Omega)}\left\|c_{h}\right\|_{L^{4}(\Omega)}\left(\sum_{e \in \Gamma_{h}} h^{-3} \int_{e}\left[w_{h}\right]^{4}\right)^{1 / 4} .
$$

We apply the same idea as in (3.21) to the last term and have:

$$
\left(w_{h}^{\text {down }} \mathbf{u}_{h} \cdot \mathbf{n}_{e},\left[c_{h}\right]\right)_{\Gamma_{h}} \lesssim\left\|\mathbf{u}_{h}\right\|_{L^{2}(\Omega)}^{1 / 2}\left\|w_{h}\right\|_{L^{4}(\Omega)}\left(\sum_{e \in \Gamma_{h}} h^{-1} \int_{e}\left(1+\left\{\left|\mathbf{u}_{h}\right|\right\}\right)\left[c_{h}\right]^{2}\right)^{1 / 2} .
$$

Therefore, the bound (3.3) is obtained by combining (3.18), (3.19), (3.20), (3.22) and (3.23).

The proof of Lemma 3.5 is now given.

Proof. We first note that

$$
B_{c q}\left(w_{h}, w_{h} ; \mathbf{u}_{h}\right)=\frac{1}{2}\left(\left(\left(q^{I}+q^{P}\right) w_{h}, w_{h}\right)+\left(\left|\mathbf{u}_{h} \cdot \mathbf{n}_{e}\right|\left[w_{h}\right],\left[w_{h}\right]\right)_{\Gamma_{h}}\right) .
$$

Next we show (3.4) by first noting:

$$
B_{d}\left(w_{h}, w_{h} ; \mathbf{u}_{h}\right)=\left(\mathbf{D}\left(\mathbf{u}_{h}\right) \nabla w_{h}, \nabla w_{h}\right)_{\mathcal{E}_{h}}+(\epsilon-1)\left(\left[w_{h}\right],\left\{\mathbf{D}\left(\mathbf{u}_{h}\right) \nabla w_{h} \cdot \mathbf{n}_{e}\right\}\right)_{\Gamma_{h}}+\left(\sigma h^{-1}\left(1+\left\{\left|\mathbf{u}_{h}\right|\right\}\right)\left[w_{h}\right],\left[w_{h}\right]\right)_{\Gamma_{h}} .
$$

We fix a face $e$ shared by $E_{+}^{e}$ and $E_{-}^{e}$ and denote by $v^{+}$(resp. $v^{-}$) the restriction of any function $v$ to $E_{+}^{e}$ (resp. $\left.E_{-}^{e}\right)$. We begin by expanding and bounding the terms using Cauchy-Schwarz's inequality,

$$
\begin{aligned}
\left(\left[w_{h}\right],\left\{\mathbf{D}\left(\mathbf{u}_{h}\right) \nabla w_{h} \cdot \mathbf{n}_{e}\right\}\right)_{e} & \lesssim\left(\left[w_{h}\right], \mathbf{D}\left(\mathbf{u}_{h}^{+}\right) \nabla w_{h}^{+} \cdot \mathbf{n}_{e}\right)_{e}+\left(\left[w_{h}\right], \mathbf{D}\left(\mathbf{u}_{h}^{-}\right) \nabla w_{h}^{-} \cdot \mathbf{n}_{e}\right)_{e} \\
& \lesssim\left\{\int_{e}\left|\mathbf{D}^{1 / 2}\left(\mathbf{u}_{h}\right) \mathbf{n}_{e}\right|\left|\left[w_{h}\right]\right|\left|\mathbf{D}^{1 / 2}\left(\mathbf{u}_{h}\right) \nabla w_{h}\right|\right\} \\
& \lesssim\left\{\left(\int_{e}\left|\mathbf{D}^{1 / 2}\left(\mathbf{u}_{h}\right) \mathbf{n}_{e}\right|^{2}\left[w_{h}\right]^{2}\right)^{1 / 2}\left(\int_{e}\left|\mathbf{D}^{1 / 2}\left(\mathbf{u}_{h}\right) \nabla w_{h}\right|^{2}\right)^{1 / 2}\right\} \\
& \lesssim\left(\int_{e}\left\{\left|\mathbf{D}^{1 / 2}\left(\mathbf{u}_{h}\right) \mathbf{n}_{e}\right|\right\}^{2}\left[w_{h}\right]^{2}\right)^{1 / 2}\left\{\left(\int_{e}\left|\mathbf{D}^{1 / 2}\left(\mathbf{u}_{h}\right) \nabla w_{h}\right|^{2}\right)^{1 / 2}\right\} .
\end{aligned}
$$

By the property (2.7), we obtain

$$
\left(\left[w_{h}\right],\left\{\mathbf{D}\left(\mathbf{u}_{h}\right) \nabla w_{h} \cdot \mathbf{n}_{e}\right\}\right)_{e} \lesssim\left(\int_{e}\left(1+\left\{\left|\mathbf{u}_{h}\right|\right\}\right)\left[w_{h}\right]^{2}\right)^{1 / 2}\left\{\left\|\mathbf{D}^{1 / 2}\left(\mathbf{u}_{h}\right) \nabla w_{h}\right\|_{L^{2}(e)}\right\} .
$$

Using a trace inequality (see (A.1) in appendix), we obtain

$$
\begin{aligned}
\left\|\mathbf{D}^{1 / 2}\left(\mathbf{u}_{h}^{+}\right) \nabla w_{h}^{+}\right\|_{L^{2}(e)} & \lesssim h^{-1 / 2}\left(\left\|\nabla w_{h}\right\|_{L^{2}\left(E_{+}^{e}\right)}^{2}+\left\|\left|\mathbf{u}_{h}\right|^{1 / 2}\left|\nabla w_{h}\right|\right\|_{L^{2}\left(E_{+}^{e}\right)}^{2}\right)^{1 / 2} \\
& \lesssim h^{-1 / 2}\left(\int_{E_{+}^{e}}\left(1+\left|\mathbf{u}_{h}\right|\right)\left|\nabla w_{h}\right|^{2}\right)^{1 / 2}
\end{aligned}
$$

Using (2.7), we have then

$$
\left\|\mathbf{D}^{1 / 2}\left(\mathbf{u}_{h}^{+}\right) \nabla w_{h}^{+}\right\|_{L^{2}(e)} \lesssim h^{-1 / 2}\left\|\mathbf{D}^{1 / 2}\left(\mathbf{u}_{h}\right) \nabla w_{h}\right\|_{L^{2}\left(E_{+}^{e}\right)} .
$$


Therefore, we have

$$
\left\{\left\|\mathbf{D}^{1 / 2}\left(\mathbf{u}_{h}\right) \nabla w_{h}\right\|_{L^{2}(e)}\right\} \lesssim h^{-1 / 2}\left\{\left\|\mathbf{D}^{1 / 2}\left(\mathbf{u}_{h}\right) \nabla w_{h}\right\|_{L^{2}\left(E^{e}\right)}\right\} .
$$

where by convention we have $\left\{\|\cdot\|_{L^{2}\left(E^{e}\right)}\right\}=0.5\|\cdot\|_{L^{2}\left(E_{+}^{e}\right)}+0.5\|\cdot\|_{L^{2}\left(E_{-}^{e}\right)}$. So, summing over the faces, we see that there is a positive constant $M$ such that

$$
\begin{gathered}
\left(\left[w_{h}\right],\left\{\mathbf{D}\left(\mathbf{u}_{h}\right) \nabla w_{h} \cdot \mathbf{n}_{e}\right\}\right)_{\Gamma_{h}}=\sum_{e \in \Gamma_{h}}\left(\left[w_{h}\right],\left\{\mathbf{D}\left(\mathbf{u}_{h}\right) \nabla w_{h} \cdot \mathbf{n}_{e}\right\}\right)_{e} \\
\lesssim \sum_{e \in \Gamma_{h}}\left(\int_{e} h^{-1}\left(1+\left\{\left|\mathbf{u}_{h}\right|\right\}\right)\left[w_{h}\right]^{2}\right)^{1 / 2}\left\{\left\|\mathbf{D}^{1 / 2}\left(\mathbf{u}_{h}\right) \nabla w_{h}\right\|_{L^{2}\left(E^{e}\right)}\right\} \\
\leq M\left(\sum_{e \in \Gamma_{h}} h^{-1}\left\|\left(1+\left\{\left|\mathbf{u}_{h}\right|\right\}\right)^{1 / 2}\left[w_{h}\right]\right\|_{L^{2}(e)}^{2}\right)^{1 / 2}\left\|\mathbf{D}^{1 / 2}\left(\mathbf{u}_{h}\right) \nabla w_{h}\right\|_{L^{2}\left(\mathcal{E}_{h}\right)} .
\end{gathered}
$$

Therefore with Young's inequality, for any positive constant, $\delta$, we have

$$
B_{d}\left(w_{h}, w_{h} ; \mathbf{u}_{h}\right) \geq\left(1+\frac{\delta}{2}(\epsilon-1)\right)\left\|\mathbf{D}^{1 / 2}\left(\mathbf{u}_{h}\right) \nabla w_{h}\right\|_{L^{2}\left(\mathcal{E}_{h}\right)}^{2}+\sum_{e \in \Gamma_{h}}\left(\sigma+\frac{\epsilon-1}{2 \delta} M^{2}\right) h^{-1}\left\|\left(1+\left\{\left|\mathbf{u}_{h}\right|\right\}\right)^{1 / 2}\left[w_{h}\right]\right\|_{L^{2}(e)}^{2} .
$$

If $\epsilon=0$, we choose $\delta=1$ and $\sigma \geq \frac{1}{2}\left(1+M^{2}\right)$. If $\epsilon=-1$, we choose $\delta=\frac{1}{2}$ and $\sigma \geq \frac{1}{2}+2 M^{2}$. Finally, if $\epsilon=1$, there is no constraint on the penalty parameter. Thus, we have proved (3.4).

\subsection{Compactness of the concentration}

One important and challenging step in proving convergence of the numerical approximation of the concentration is to show compactness of $\left\{c_{h}\right\}_{h>0}$. This is stated in the following theorem, which is a non-trivial application of Theorem 3.2.

Theorem 3.7. Suppose the maximal time step $\Delta t$ tends to zero with the mesh parameter $h$. Then the concentration $\left\{c_{h}\right\}_{h>0}$ computed using the numerical scheme (2.10) are precompact in $L^{2}\left[0, T ; L^{2}(\Omega)\right] \cap L^{r}\left[0, T ; W^{1,4}(\Omega)^{\prime}\right]$ for all $1 \leq r<\infty$.

Proof. We apply Theorem 3.2 with the following choice of spaces:

$$
W=W^{1,4}(\Omega), \quad V=B V(\Omega) \cap L^{4}(\Omega), \quad H=L^{2}(\Omega), \quad W\left(\mathcal{E}_{h}\right)=W^{1,4}\left(\mathcal{E}_{h}\right), \quad W_{h}=C_{h} .
$$

The spaces $W, V$ and $H$ are clearly Banach spaces and it is easy to check that $W\left(\mathcal{E}_{h}\right)$ equipped with the following norm is a Banach space.

$$
\|w\|_{W\left(\mathcal{E}_{h}\right)}=\|w\|_{W^{1,4}\left(\mathcal{E}_{h}\right)} .
$$

From $[1,17]$, we also have that $W \subset H$ is dense and $W \hookrightarrow V \hookrightarrow H \hookrightarrow W^{\prime}$ are dense embeddings with $V$ compactly embedded in $H$. Next we easily see that $W^{1,4}(\Omega)$ is embedded in $W^{1,4}\left(\mathcal{E}_{h}\right)$, which is itself embedded in $V$, with embedding constants independent of $h$. It remains to check the assumptions of Theorem 3.2. The fact that the $L^{2}$ projection, $\Pi_{h}: L^{2}(\Omega) \rightarrow C_{h}$, is stable in $W^{1,4}\left(\mathcal{E}_{h}\right)$ is proved in Lemma 3.8. Assumption 1 in Theorem 3.2 is immediately satisfied if the inner-product on $H$ is the weighted $L^{2}$ inner-product with weight $\phi$ and if we define the function $F_{h}$ as:

$$
F_{h}\left(w_{h}\right)=\left(\hat{c} q^{I}, w_{h}\right)-B_{d}\left(c_{h}, w_{h} ; \mathbf{u}_{h}\right)-B_{c q}\left(c_{h}, w_{h} ; \mathbf{u}_{h}\right) .
$$

Assumption 2 is satisfied for $p=2$, since the boundedness of $\left\{c_{h}\right\}_{h>0}$ in $L^{2}(0, T ; V)$ is a consequence of the embedding of $H^{1}\left(\mathcal{E}_{h}\right)$ into $V$ and the boundedness of $\left\{\left\|c_{h}\right\|_{L^{2}\left(0, T ; H^{1}\left(\mathcal{E}_{h}\right)\right)}\right\}_{h>0}$. Finally, it remains to check 
Assumption 3 of Theorem 3.2. This requires upper bounds for the forms $B_{d}, B_{c q}$, that are proved in Lemma 3.4. Since $\hat{c} \in L^{\infty}(\Omega)$, one can easily obtain

$$
\left(\hat{c} q^{I}, w_{h}\right) \lesssim\left\|q^{I}\right\|_{L^{2}(\Omega)}\left\|w_{h}\right\|_{L^{4}(\Omega)} .
$$

Therefore, by Lemma 3.4 we have,

$$
\left|F_{h}\left(w_{h}\right)\right| \leq M\left\|w_{h}\right\|_{W^{1,4}\left(\mathcal{E}_{h}\right)}\left(\left(1+\left\|\mathbf{u}_{h}\right\|_{L^{2}(\Omega)}^{1 / 2}\right)\left\|c_{h}\right\|_{C_{h}}+\left\|q^{I}\right\|_{L^{2}(\Omega)}+\left(\left\|q^{I}+q^{P}\right\|_{L^{2}(\Omega)}+\left\|\mathbf{u}_{h}\right\|_{L^{2}(\Omega)}\right)\left\|c_{h}\right\|_{L^{4}(\Omega)}\right),
$$

with the constant $M$ independent of the mesh size.

From [1], [5, 6, 8] and (2.7),

$$
\left\|c_{h}\right\|_{L^{4}(\Omega)} \lesssim\left\|c_{h}\right\|_{H^{1}\left(\mathcal{E}_{h}\right)}
$$

Hence, using Cauchy-Schwarz's inequality

$$
\begin{aligned}
\int_{0}^{T}\left|F_{h}\left(w_{h}\right)\right| \leq & M \int_{0}^{T}\left\|w_{h}\right\|_{W^{1,4}\left(\mathcal{E}_{h}\right)}\left(\left(1+\left\|\mathbf{u}_{h}\right\|_{L^{2}(\Omega)}^{1 / 2}\right)\left\|c_{h}\right\|_{C_{h}}+\left\|q^{I}\right\|_{L^{2}(\Omega)}\right. \\
& \left.+\left(\left\|q^{I}+q^{P}\right\|_{L^{2}(\Omega)}+\left\|\mathbf{u}_{h}\right\|_{L^{2}(\Omega)}\right)\left\|c_{h}\right\|_{L^{4}(\Omega)}\right) \\
\leq & M\left(\left(1+\left\|\mathbf{u}_{h}\right\|_{L^{\infty}\left[0, T ; L^{2}(\Omega)\right]}^{1 / 2}\right)\left\|c_{h}\right\|_{L^{2}\left[0, T ; C_{h}\right]}\right. \\
& +\left\|q^{I}\right\|_{L^{\infty}\left[0, T ; L^{2}(\Omega)\right]}+\left(\left\|q^{I}+q^{P}\right\|_{L^{\infty}\left[0, T ; L^{2}(\Omega)\right]}\right. \\
& \left.\left.+\left\|\mathbf{u}_{h}\right\|_{L^{\infty}\left[0, T ; L^{2}(\Omega)\right]}\right)\left\|c_{h}\right\|_{L^{2}\left[0, T ; H^{1}\left(\mathcal{E}_{h}\right)\right]}\right)\left\|w_{h}\right\|_{L^{4}\left[0, T ; W^{1,4}\left(\mathcal{E}_{h}\right)\right]}
\end{aligned}
$$

Therefore, $F_{h}$ belongs to $L^{1}\left[0, T ; W_{h}^{\prime}\right]$ and we have

$$
\begin{aligned}
\left\|F_{h}\right\|_{L^{1}\left[0, T ; W_{h}^{\prime}\right]} \leq & M\left(\left(1+\left\|\mathbf{u}_{h}\right\|_{L^{\infty}\left[0, T ; L^{2}(\Omega)\right]}^{1 / 2}\right)\left\|c_{h}\right\|_{L^{2}\left[0, T ; C_{h}\right]}+\left\|q^{I}\right\|_{L^{\infty}\left[0, T ; L^{2}(\Omega)\right]}\right. \\
& \left.+\left(\left\|q^{I}+q^{P}\right\|_{L^{\infty}\left[0, T ; L^{2}(\Omega)\right]}+\left\|\mathbf{u}_{h}\right\|_{L^{\infty}\left[0, T ; L^{2}(\Omega)\right]}\right)\left\|c_{h}\right\|_{L^{2}\left[0, T ; H^{1}\left(\mathcal{E}_{h}\right)\right]}\right) .
\end{aligned}
$$

Furthermore, according to the stability analysis in Theorems 3.3 and 3.6 , we know that $\left\|\mathbf{u}_{h}\right\|_{L^{\infty}\left[0, T ; L^{2}(\Omega)\right]}$, $\left\|c_{h}\right\|_{L^{2}\left[0, T ; H^{1}\left(\mathcal{E}_{h}\right)\right]}$ and $\left\|c_{h}\right\|_{L^{2}\left[0, T ; C_{h}\right]}$ are bounded by a constant independent of $h$ and $\Delta t$. Therefore, $\left\{\left\|F_{h}\right\|_{L^{1}\left[0, T, W_{h}^{\prime}\right]}\right\}_{h>0}$ is bounded.

Lemma 3.8. The $L^{2}$ projection

$$
\Pi_{h}: L^{2}(\Omega) \rightarrow C_{h}
$$

is stable in $W\left(\mathcal{E}_{h}\right)=W^{1,4}\left(\mathcal{E}_{h}\right)$, i.e. there is a constant $M>0$ independent of $h$ such that

$$
\left\|\Pi_{h} w\right\|_{W\left(\mathcal{E}_{h}\right)} \leq M\|w\|_{W\left(\mathcal{E}_{h}\right)}, \quad \forall w \in W\left(\mathcal{E}_{h}\right) .
$$

Proof. Fix $w \in W^{1,4}\left(\mathcal{E}_{h}\right)$. For the term $\left\|\Pi_{h} w\right\|_{L^{4}(\Omega)}$, we use an inverse inequality, the stability of $\Pi_{h}$ in $L^{2}$ and Cauchy-Schwarz's inequality to obtain

$$
\left\|\Pi_{h} w\right\|_{L^{4}(\Omega)}^{4}=\sum_{E \in \mathcal{E}_{h}}\left\|\Pi_{h} w\right\|_{L^{4}(E)}^{4} \lesssim \sum_{E \in \mathcal{E}_{h}} h^{-d}\left\|\Pi_{h} w\right\|_{L^{2}(E)}^{4} \lesssim \sum_{E \in \mathcal{E}_{h}} h^{-d}\|w\|_{L^{2}(E)}^{4} \lesssim\|w\|_{L^{4}(\Omega)}^{4}
$$

Next, let $\bar{w}$ denote the average of $w$ on each element, i.e.

$$
\left.\bar{w}\right|_{E}=\frac{1}{|E|} \int_{E} w, \quad \forall E \in \mathcal{E}_{h} .
$$


Thus, we have

$$
\begin{array}{r}
\left(\sum_{E \in \mathcal{E}_{h}}\left\|\nabla \Pi_{h} w\right\|_{L^{4}(E)}^{4}+\sum_{e \in \Gamma_{h}} h^{-3}\left\|\left[\Pi_{h} w\right]\right\|_{L^{4}(e)}^{4}\right)^{1 / 4} \leq\left(\sum_{e \in \Gamma_{h}} h^{-3}\left\|\left[\Pi_{h} \bar{w}\right]\right\|_{L^{4}(e)}^{4}\right)^{1 / 4} \\
+\left(\sum_{E \in \mathcal{E}_{h}}\left\|\nabla \Pi_{h}(w-\bar{w})\right\|_{L^{4}(E)}^{4}+\sum_{e \in \Gamma_{h}} h^{-3}\left\|\left[\Pi_{h}(w-\bar{w})\right]\right\|_{L^{4}(e)}^{4}\right)^{1 / 4} .
\end{array}
$$

For the first term in the upper bound, we have

$$
\left\|\left[\Pi_{h} \bar{w}\right]\right\|_{L^{4}(e)}=\|[\bar{w}]\|_{L^{4}(e)} \leq\|[w-\bar{w}]\|_{L^{4}(e)}+\|[w]\|_{L^{4}(e)} .
$$

From [2], we have

$$
\sum_{e \in \Gamma_{h}} h^{-3}\|[w-\bar{w}]\|_{L^{4}(e)}^{4} \lesssim \sum_{E \in \mathcal{E}_{h}}\|\nabla w\|_{L^{4}(E)}^{4} .
$$

Hence, we have

$$
\sum_{e \in \Gamma_{h}} h^{-3}\left\|\left[\Pi_{h} \bar{w}\right]\right\|_{L^{4}(e)}^{4} \lesssim \sum_{E \in \mathcal{E}_{h}}\|\nabla w\|_{L^{4}(E)}^{4}+\sum_{e \in \Gamma_{h}} h^{-3}\|[w]\|_{L^{4}(e)}^{4} .
$$

Using the same derivation as in (3.30), we have:

$$
\sum_{E \in \mathcal{E}_{h}}\left\|\nabla \Pi_{h}(w-\bar{w})\right\|_{L^{4}(E)}^{4}=\sum_{E \in \mathcal{E}_{h}}\left\|\nabla \Pi_{h} w\right\|_{L^{4}(E)}^{4} \lesssim \sum_{E \in \mathcal{E}_{h}}\|\nabla w\|_{L^{4}(E)}^{4} .
$$

Furthermore, by trace and inverse inequalities we obtain

$$
\begin{aligned}
\left\|\Pi_{h}(w-\bar{w})\right\|_{L^{4}(e)} & \leq M h_{E}^{-1 / 4}\left\|\Pi_{h}(w-\bar{w})\right\|_{L^{4}(E)} \leq M h_{E}^{-1 / 4} h_{E}^{-d / 4}\left\|\Pi_{h}(w-\bar{w})\right\|_{L^{2}(E)} \\
& \leq M h_{E}^{-1 / 4} h_{E}^{-d / 4}\|w-\bar{w}\|_{L^{2}(E)} \leq M h_{E}^{1 / 4} h_{E}^{-d / 4} h_{E}^{-1 / 2}\|w-\bar{w}\|_{L^{2}(E)} \\
& \leq M h_{E}^{1 / 4} h_{E}^{-d / 4} h_{E}^{1 / 2}\|\nabla w\|_{L^{2}(E)} \leq M h_{E}^{1 / 4} h_{E}^{-d / 4} h_{E}^{1 / 2} h_{E}^{d / 4}\|\nabla w\|_{L^{4}(E)} \\
& \leq M h^{3 / 4}\|\nabla w\|_{L^{4}(E)} .
\end{aligned}
$$

Hence, we have

$$
\sum_{e \in \Gamma_{h}} h^{-3}\left\|\left[\Pi_{h}(w-\bar{w})\right]\right\|_{L^{4}(e)}^{4} \leq M \sum_{E \in \mathcal{E}_{h}}\|\nabla w\|_{L^{4}(E)}^{4} .
$$

So, we can conclude by combining all the bounds above.

\subsection{Convergence of the solutions}

From Theorem 3.7 and the precompactness of $\left\{c_{h}\right\}_{h>0}$ in $L^{2}\left[0, T ; L^{2}(\Omega)\right]$, there exists a subsequence, still denoted by $\left\{c_{h}\right\}_{h>0}$ that converges strongly in $L^{2}\left[0, T ; L^{2}(\Omega)\right]$ to a function $c \in L^{2}\left[0, T ; L^{2}(\Omega)\right]$. This is sufficient to show strong convergence of the numerical approximations of velocity and pressure.

Theorem 3.9. Given the data, parameters and numerical scheme, and suppose the maximal time step $\Delta t$ tends to zero with the mesh parameter. Suppose that the sequence $\left\{c_{h}\right\}_{h>0} \subset L^{2}\left[0, T ; L^{2}(\Omega)\right]$ converges to $c$ in $L^{2}\left[0, T ; L^{2}(\Omega)\right]$, then the velocity and pressure computed using the scheme (2.8)-(2.9) over the regular family of meshes converge strongly to the solutions $(\mathbf{u}, p)$ of the weak forms (2.4) and (2.5).

The proof of this result is skipped as it follows closely the proof of Theorem 3.10 in [17]. 
Theorem 3.10. Suppose that the maximal time step $\Delta t$ and $h$ tend to zero with mesh parameter. Then upon passage to a subsequence, the concentrations $\left\{c_{h}\right\}_{h}$ computed using the scheme (2.10) with SIPG namely with $\epsilon=-1$ over a regular family of meshes converge strongly in $L^{2}\left[0, T ; L^{2}(\Omega)\right]$ to $c \in L^{2}\left[0, T ; H^{1}(\Omega)\right]$, that satisfies the weak formulation (2.6).

Proof. The uniform boundedness of $\left\{\left\|c_{h}\right\|_{L^{2}\left[0, T ; H^{1}\left(\mathcal{E}_{h}\right)\right]}\right\}_{h>0}$, obtained from Theorem 3.6, implies that every accumulation point of $\left\{c_{h}\right\}_{h>0}$ in $L^{2}\left[0, T ; L^{2}(\Omega)\right]$ belongs to $L^{2}\left[0, T ; H^{1}(\Omega)\right]$, and that there exists a subsequence, still denoted by $\left\{c_{h}\right\}_{h>0}$, such that $\left\{\nabla c_{h}\right\}_{h>0}$ converges weakly in $L^{2}\left[0, T ; H^{-1}(\Omega)\right]$ to $\nabla c$ (see Thm. 7.1 in [1]). Let $w \in C^{\infty}\left[0, T ; C^{\infty}(\Omega)\right]$ and $w(T)=0$. Approximation theory guarantees existence of $w_{h} \in C\left[0, T ; L^{2}(\Omega)\right]$ such that $\left.w_{h}\right|_{\left(t^{n-1}, t^{n}\right)}$ belongs to $\mathcal{P}_{\ell}\left[t^{n-1}, t^{n} ; C_{h}\right]$, with $w_{h}(T)=0$ and such that the sequence $\left\{w_{h}\right\}_{h>0}$ converges strongly to $w$ in the following sense

$$
\begin{gathered}
\lim _{h \rightarrow 0}\left\|w_{h}-w\right\|_{L^{\infty}\left[0, T ; L^{\infty}(\Omega)\right]}=0, \\
\lim _{h \rightarrow 0}\left\|\nabla w_{h}-\nabla w\right\|_{L^{\infty}\left[0, T ; L^{\infty}(\Omega)\right]}=0 .
\end{gathered}
$$

Integrating the temporal term in (2.10), summing over $n$, and using the fact that $w_{h}(T)=0$, yields

$$
\int_{0}^{T}\left(-\left(\phi c_{h}, \partial_{t} w_{h}\right)+B_{d}\left(c_{h}, w_{h} ; \mathbf{u}_{h}\right)+B_{c q}\left(c_{h}, w_{h} ; \mathbf{u}_{h}\right)\right)=\left(\phi c_{h-}^{0}, w_{h}(0)\right)+\int_{0}^{T}\left(\hat{c} q^{I}, w_{h}\right) .
$$

We now pass to the limit term by term in (3.32). We clearly have

$$
\begin{array}{r}
\lim _{h \rightarrow 0} \int_{0}^{T}\left(\phi c_{h}, \partial_{t} w_{h}\right)=\int_{0}^{T}\left(\phi c, \partial_{t} w\right), \\
\lim _{h \rightarrow 0}\left(\phi c_{h-}^{0}, w_{h}(0)\right)=\left(\phi c_{0}, w(0)\right), \\
\lim _{h \rightarrow 0} \int_{0}^{T}\left(\hat{c} q^{I}, w_{h}\right)=\int_{0}^{T}\left(\hat{c} q^{I}, w\right) .
\end{array}
$$

Next we will show that

$$
\int_{0}^{T}(\nabla c, \mathbf{D}(\mathbf{u}) \nabla w)=\lim _{h \rightarrow 0} \int_{0}^{T} B_{d}\left(c_{h}, w_{h} ; \mathbf{u}_{h}\right) .
$$

The proof of this result is technical and requires the introduction of two operators, $\mathbf{G}$ and $\mathbf{D}_{h}$. The approximate gradient $\mathbf{G} c_{h} \in L^{2}\left[0, T ; \mathcal{P}_{2 r-1}\left(\mathcal{E}_{h}\right)^{d}\right]$ is defined by

$$
\left(\mathbf{G} c_{h}(t), \mathbf{a}\right)=\left(\nabla c_{h}(t), \mathbf{a}\right)_{\mathcal{E}_{h}}-\left(\left[c_{h}(t)\right],\left\{\mathbf{a} \cdot \mathbf{n}_{e}\right\}\right)_{\Gamma_{h}}, \quad \forall \mathbf{a} \in \mathcal{P}_{2 r-1}\left(\mathcal{E}_{h}\right)^{d}, \quad \forall t .
$$

The uniform boundedness of $\left\{\left\|c_{h}\right\|_{L^{2}\left[0, T ; H^{1}\left(\mathcal{E}_{h}\right)\right]}\right\}_{h>0}$ implies that the subsequence $\left\{\mathbf{G} c_{h}\right\}_{h>0}$ converges weakly to $\nabla c$ in $L^{2}\left[0, T ; L^{2}(\Omega)\right]$ (see Thm. 6.3 in [1]).

The matrix $\mathbf{D}_{h}(\mathbf{v})$ is a piecewise constant matrix defined by

$$
\left.\mathbf{D}_{h}(\mathbf{v})\right|_{E}=\mathbf{D}\left(\left.\tilde{\mathbf{v}}\right|_{E}\right),\left.\quad \tilde{\mathbf{v}}\right|_{E}=\frac{1}{|E|} \int_{E} \mathbf{v}, \quad \forall E \in \mathcal{E}_{h} .
$$

By the Lipschitz continuity of the diffusion-dispersion tensor $\mathbf{D}$, we have

$$
\begin{gathered}
\left\|\mathbf{D}_{h}\left(\mathbf{u}_{h}\right)-\mathbf{D}(\mathbf{u})\right\|_{L^{2}(\Omega)}=\left\|\mathbf{D}\left(\tilde{\mathbf{u}}_{h}\right)-\mathbf{D}(\mathbf{u})\right\|_{L^{2}(\Omega)} \lesssim\left\|\tilde{\mathbf{u}}_{h}-\mathbf{u}\right\|_{L^{2}(\Omega)} \\
\lesssim\left\|\tilde{\mathbf{u}}_{h}-\tilde{\mathbf{u}}\right\|_{L^{2}(\Omega)}+\|\tilde{\mathbf{u}}-\mathbf{u}\|_{L^{2}(\Omega)}
\end{gathered}
$$


Since $\tilde{\mathbf{u}}$ is the piecewise constant approximation of $\mathbf{u}$, then

$$
\lim _{h \rightarrow 0} \int_{0}^{T}\|\tilde{\mathbf{u}}-\mathbf{u}\|_{L^{2}(\Omega)}^{2}=0
$$

Furthermore,

$$
\left\|\tilde{\mathbf{u}}_{h}-\tilde{\mathbf{u}}\right\|_{L^{2}(\Omega)} \leq\left\|\mathbf{u}_{h}-\mathbf{u}\right\|_{L^{2}(\Omega)} .
$$

Since the sequence $\left\{\mathbf{u}_{h}\right\}_{h}$ converges strongly to $\mathbf{u}$ in $L^{2}\left[0, T ; L^{2}(\Omega)\right]$, we have

$$
\lim _{h \rightarrow 0} \int_{0}^{T}\left\|\tilde{\mathbf{u}}_{h}-\tilde{\mathbf{u}}\right\|_{L^{2}(\Omega)}^{2}=0 .
$$

Therefore, we can conclude

$$
\lim _{h \rightarrow 0} \int_{0}^{T}\left\|\mathbf{D}_{h}\left(\mathbf{u}_{h}\right)-\mathbf{D}(\mathbf{u})\right\|_{L^{2}(\Omega)}^{2}=0 .
$$

Since we also have the property,

$$
\lim _{h \rightarrow 0} \int_{0}^{T}\left\|\mathbf{D}\left(\mathbf{u}_{h}\right)-\mathbf{D}(\mathbf{u})\right\|_{L^{2}(\Omega)}^{2}=0
$$

Consequently we have

$$
\lim _{h \rightarrow 0} \int_{0}^{T}\left\|\mathbf{D}\left(\mathbf{u}_{h}\right)-\mathbf{D}_{h}\left(\mathbf{u}_{h}\right)\right\|_{L^{2}(\Omega)}^{2}=0
$$

From the property (3.38), we have

$$
\begin{aligned}
\lim _{h \rightarrow 0} \int_{0}^{T}\left(\nabla c_{h}, \mathbf{D}\left(\mathbf{u}_{h}\right) \nabla w_{h}\right) \mathcal{E}_{h} & =\lim _{h \rightarrow 0} \int_{0}^{T}\left(\nabla c_{h},\left(\mathbf{D}\left(\mathbf{u}_{h}\right)-\mathbf{D}_{h}\left(\mathbf{u}_{h}\right)\right) \nabla w_{h}\right)_{\mathcal{E}_{h}}+\lim _{h \rightarrow 0} \int_{0}^{T}\left(\nabla c_{h}, \mathbf{D}_{h}\left(\mathbf{u}_{h}\right) \nabla w_{h}\right)_{\mathcal{E}_{h}} \\
& =\lim _{h \rightarrow 0} \int_{0}^{T}\left(\nabla c_{h}, \mathbf{D}_{h}\left(\mathbf{u}_{h}\right) \nabla w_{h}\right) \mathcal{E}_{h} .
\end{aligned}
$$

Additionally from the property (3.36) and (3.31), we have

$$
\int_{0}^{T}(\nabla c, \mathbf{D}(\mathbf{u}) \nabla w)=\lim _{h \rightarrow 0} \int_{0}^{T}\left(\nabla c, \mathbf{D}_{h}\left(\mathbf{u}_{h}\right) \nabla w_{h}\right)_{\mathcal{E}_{h}} .
$$

We also observe,

$$
\begin{aligned}
\int_{0}^{T} \mid\left(\mathbf{G} c_{h}, \mathbf{D}_{h}\left(\mathbf{u}_{h}\right)\right. & \left.\nabla w_{h}\right)_{\mathcal{E}_{h}}-\left(\nabla c, \mathbf{D}_{h}\left(\mathbf{u}_{h}\right) \nabla w_{h}\right) \mathcal{E}_{h}\left|\leq \int_{0}^{T}\right|\left(\mathbf{G} c_{h}-\nabla c, \mathbf{D}(\mathbf{u}) \nabla w\right)_{\mathcal{E}_{h}} \mid \\
& +\int_{0}^{T}\left|\left(\mathbf{G} c_{h},\left(\mathbf{D}_{h}\left(\mathbf{u}_{h}\right)-\mathbf{D}(\mathbf{u})\right) \nabla w_{h}\right)_{\mathcal{E}_{h}}\right|+\left|\left(\mathbf{G} c_{h}, \mathbf{D}(\mathbf{u})\left(\nabla w_{h}-\nabla w\right)\right)_{\mathcal{E}_{h}}\right| \\
& +\int_{0}^{T}\left|\left(\nabla c,\left(\mathbf{D}(\mathbf{u})-\mathbf{D}_{h}\left(\mathbf{u}_{h}\right)\right) \nabla w\right)_{\mathcal{E}_{h}}\right|+\left|\left(\nabla c, \mathbf{D}_{h}\left(\mathbf{u}_{h}\right)\left(\nabla w-\nabla w_{h}\right)\right)_{\mathcal{E}_{h}}\right| .
\end{aligned}
$$

Therefore, we have from (3.31), (3.36) and the weak convergence of $\left\{\mathbf{G} c_{h}\right\}_{h}$ to $\nabla c$

$$
\lim _{h \rightarrow 0} \int_{0}^{T}\left(\nabla c, \mathbf{D}_{h}\left(\mathbf{u}_{h}\right) \nabla w_{h}\right)_{\mathcal{E}_{h}}=\lim _{h \rightarrow 0} \int_{0}^{T}\left(\mathbf{G} c_{h}, \mathbf{D}_{h}\left(\mathbf{u}_{h}\right) \nabla w_{h}\right)_{\mathcal{E}_{h}} .
$$


Thus, we conclude with (3.40), (3.41) and (3.34)

$$
\begin{aligned}
\int_{0}^{T}(\nabla c, \mathbf{D}(\mathbf{u}) \nabla w) & =\lim _{h \rightarrow 0} \int_{0}^{T}\left(\nabla c, \mathbf{D}_{h}\left(\mathbf{u}_{h}\right) \nabla w_{h}\right)_{\mathcal{E}_{h}}=\lim _{h \rightarrow 0} \int_{0}^{T}\left(\mathbf{G} c_{h}, \mathbf{D}_{h}\left(\mathbf{u}_{h}\right) \nabla w_{h}\right)_{\mathcal{E}_{h}} \\
& =\lim _{h \rightarrow 0} \int_{0}^{T}\left(\left(\nabla c_{h}, \mathbf{D}_{h}\left(\mathbf{u}_{h}\right) \nabla w_{h}\right)_{\mathcal{E}_{h}}-\left(\left[c_{h}\right],\left\{\mathbf{D}_{h}\left(\mathbf{u}_{h}\right) \nabla w_{h} \cdot \mathbf{n}_{e}\right\}\right)_{\Gamma_{h}}\right) .
\end{aligned}
$$

Using a trace inequality, we write

$$
\begin{gathered}
\int_{0}^{T}\left(\left[c_{h}\right],\left\{\left(\mathbf{D}\left(\mathbf{u}_{h}\right)-\mathbf{D}_{h}\left(\mathbf{u}_{h}\right)\right) \nabla w_{h} \cdot \mathbf{n}_{e}\right\}\right)_{\Gamma_{h}} \lesssim\left\|c_{h}\right\|_{C_{h}}\left(\sum_{e \in \Gamma_{h}} h\left\|\mathbf{D}\left(\mathbf{u}_{h}\right)-\mathbf{D}_{h}\left(\mathbf{u}_{h}\right)\right\|_{L^{2}(e)}^{2}\right)^{1 / 2}\left\|\nabla w_{h}\right\|_{L^{\infty}(\Omega)} \\
\lesssim\left\|c_{h}\right\|_{C_{h}}\left\|\mathbf{u}_{h}-\tilde{\mathbf{u}}_{h}\right\|_{L^{2}(\Omega)}\left(\|\nabla w\|_{L^{\infty}(\Omega)}+\left\|\nabla w-\nabla w_{h}\right\|_{L^{\infty}(\Omega)}\right) .
\end{gathered}
$$

From the stability of $c_{h}$ in $L^{2}\left[0, T ; C_{h}\right]$ and $(3.31)$, we obtain

$$
\lim _{h \rightarrow 0} \int_{0}^{T}\left(\left[c_{h}\right],\left\{\mathbf{D}\left(\mathbf{u}_{h}\right) \nabla w_{h} \cdot \mathbf{n}_{e}\right\}\right)_{\Gamma_{h}}=\lim _{h \rightarrow 0} \int_{0}^{T}\left(\left[c_{h}\right],\left\{\mathbf{D}_{h}\left(\mathbf{u}_{h}\right) \nabla w_{h} \cdot \mathbf{n}_{e}\right\}\right)_{\Gamma_{h}} .
$$

Thus (3.39), (3.42) and (3.43) imply

$$
\int_{0}^{T}(\nabla c, \mathbf{D}(\mathbf{u}) \nabla w)=\lim _{h \rightarrow 0} \int_{0}^{T}\left(\nabla c_{h}, \mathbf{D}\left(\mathbf{u}_{h}\right) \nabla w_{h}\right)_{\mathcal{E}_{h}}-\left(\left[c_{h}\right],\left\{\mathbf{D}\left(\mathbf{u}_{h}\right) \nabla w_{h} \cdot \mathbf{n}_{e}\right\}\right)_{\Gamma_{h}} .
$$

Next, let us examine the term $\left(\left[w_{h}\right],\left\{\mathbf{D}\left(\mathbf{u}_{h}\right) \nabla c_{h} \cdot \mathbf{n}_{e}\right\}\right)_{\Gamma_{h}}$. Using (3.11) and (3.6), we have

$$
\int_{0}^{T}\left(\left[w_{h}\right],\left\{\mathbf{D}\left(\mathbf{u}_{h}\right) \nabla c_{h} \cdot \mathbf{n}_{e}\right\}\right)_{\Gamma_{h}} \lesssim\left(\int_{0}^{T} \sum_{e \in \Gamma_{h}} \int_{e} h^{-1}\left(1+\left\{\left|\mathbf{u}_{h}\right|\right\}\right)\left[w_{h}\right]^{2}\right)^{1 / 2}
$$

Then, with (3.12) and (3.13), we have

$$
\int_{0}^{T}\left(\left[w_{h}\right],\left\{\mathbf{D}\left(\mathbf{u}_{h}\right) \nabla c_{h} \cdot \mathbf{n}_{e}\right\}\right)_{\Gamma_{h}} \lesssim\left(\int_{0}^{T}\left(1+\left\|\mathbf{u}_{h}\right\|_{L^{2}(\Omega)}^{2}\right)\right)^{1 / 4}\left(\int_{0}^{T} \sum_{e \in \Gamma_{h}} h^{-3}\left\|\left[w_{h}\right]\right\|_{L^{4}(e)}^{4}\right)^{1 / 4} .
$$

From Lemma 3.3 and an inverse inequality we have

$$
\int_{0}^{T}\left(\left[w_{h}\right],\left\{\mathbf{D}\left(\mathbf{u}_{h}\right) \nabla c_{h} \cdot \mathbf{n}_{e}\right\}\right)_{\Gamma_{h}} \lesssim\left(\int_{0}^{T} h^{-(2+d)} \sum_{e \in \Gamma_{h}}\left\|\left[w_{h}\right]\right\|_{L^{2}(e)}^{4}\right)^{1 / 4}
$$

We now apply Jensen's inequality and an approximation result

$$
\begin{aligned}
\int_{0}^{T}\left(\left[w_{h}\right],\left\{\mathbf{D}\left(\mathbf{u}_{h}\right) \nabla c_{h} \cdot \mathbf{n}_{e}\right\}\right)_{\Gamma_{h}} & \lesssim\left(\int_{0}^{T} h^{-(2+d)}\left(\sum_{e \in \Gamma_{h}}\left\|\left[w_{h}\right]\right\|_{L^{2}(e)}^{2}\right)^{2}\right)^{1 / 4} \\
& \lesssim\left(\int_{0}^{T} h^{-(2+d)}\left(\sum_{e \in \Gamma_{h}}\left\|\left[w_{h}-w\right]\right\|_{L^{2}(e)}^{2}\right)^{2}\right)^{1 / 4} \\
& \lesssim\left(\int_{0}^{T} h^{4-d}\|w\|_{H^{2}(\Omega)}^{4}\right)^{1 / 4} \lesssim h^{1 / 4}\left(\int_{0}^{T}\|w\|_{H^{2}(\Omega)}^{4}\right)^{1 / 4}
\end{aligned}
$$


Therefore, we have

$$
\lim _{h \rightarrow 0} \int_{0}^{T}\left(\left[w_{h}\right],\left\{\mathbf{D}\left(\mathbf{u}_{h}\right) \nabla c_{h} \cdot \mathbf{n}_{e}\right\}\right)_{\Gamma_{h}}=0 .
$$

For the penalty term, we use a similar argument

$$
\begin{aligned}
& \int_{0}^{T}\left(\sigma h^{-1}\left(1+\left\{\left|\mathbf{u}_{h}\right|\right\}\right)\left[c_{h}\right],\left[w_{h}\right]\right)_{\Gamma_{h}} \\
& \lesssim\left(\int_{0}^{T}\left(h^{-1}\left(1+\left\{\left|\mathbf{u}_{h}\right|\right\}\right)\left[w_{h}\right],\left[w_{h}\right]\right)_{\Gamma_{h}}\right)^{1 / 2}\left(\int_{0}^{T}\left\|c_{h}\right\|_{C_{h}}^{2}\right)^{1 / 2} \lesssim h^{1 / 4}\left(\int_{0}^{T}\|w\|_{H^{2}(\Omega)}^{4}\right)^{1 / 4} .
\end{aligned}
$$

Therefore, we have

$$
\lim _{h \rightarrow 0} \int_{0}^{T}\left(\sigma h^{-1}\left(1+\left\{\left|\mathbf{u}_{h}\right|\right\}\right)\left[c_{h}\right],\left[w_{h}\right]\right)_{\Gamma_{h}}=0 .
$$

Combining the results above, namely (3.44)-(3.46), yields (3.33). Next we will show that

$$
\frac{1}{2} \int_{0}^{T}\left((\mathbf{u} \cdot \nabla c, w)-(c \mathbf{u}, \nabla w)+\left(\left(q^{I}+q^{P}\right) c, w\right)\right)=\lim _{h \rightarrow 0} \int_{0}^{T} B_{c q}\left(c_{h}, w_{h} ; \mathbf{u}_{h}\right) .
$$

Since $\left\{\mathbf{u}_{h}\right\}_{h}$ converges strongly to $\mathbf{u}$ in $L^{2}\left[0, T ; L^{2}(\Omega)\right]$, it is easy to show that

$$
\frac{1}{2} \int_{0}^{T}\left(-(c \mathbf{u}, \nabla w)+\left(\left(q^{I}+q^{P}\right) c, w\right)\right)=\lim _{h \rightarrow 0} \frac{1}{2} \int_{0}^{T}\left(-\left(c_{h} \mathbf{u}_{h}, \nabla w_{h}\right)+\left(\left(q^{I}+q^{P}\right) c_{h}, w_{h}\right)\right) .
$$

Using trace inequality and inverse inequality, we also have,

$$
\begin{aligned}
\left(c_{h}^{\mathrm{up}} \mathbf{u}_{h} \cdot \mathbf{n}_{e},\left[w_{h}\right]\right)_{\Gamma_{h}} & \lesssim\left\|\mathbf{u}_{h}\right\|_{L^{2}(\Omega)}\left\|c_{h}\right\|_{L^{4}(\Omega)}\left(\sum_{e \in \Gamma_{h}} h^{-\frac{d+1}{2}} \int_{e}\left[w_{h}-w\right]^{2}\right)^{1 / 2} \\
& \lesssim h^{1 / 2}\|w\|_{H^{2}(\Omega)}\left\|\mathbf{u}_{h}\right\|_{L^{2}(\Omega)}\left\|c_{h}\right\|_{L^{4}(\Omega)} .
\end{aligned}
$$

With the stability bounds on $\mathbf{u}_{h}$ and $c_{h}$, we then have

$$
\lim _{h \rightarrow 0} \int_{0}^{T}\left(c_{h}^{\mathrm{up}} \mathbf{u}_{h} \cdot \mathbf{n}_{e},\left[w_{h}\right]\right)_{\Gamma_{h}}=0
$$

Integrating by parts on each element and summing over all elements yields:

$$
\left(c_{h}, \operatorname{div}\left(\mathbf{u}_{h} w\right)\right)=-\left(\mathbf{u}_{h} \cdot \nabla c_{h}, w\right)_{\mathcal{E}_{h}}+\left(w \mathbf{u}_{h} \cdot \mathbf{n}_{e},\left[c_{h}\right]\right)_{\Gamma_{h}} .
$$

We write

$$
\left(c_{h}, \operatorname{div}\left(\mathbf{u}_{h} w\right)\right)-(c, \operatorname{div}(\mathbf{u} w))=\left(c_{h},\left(\operatorname{div}\left(\mathbf{u}_{h}\right)-\operatorname{div}(\mathbf{u})\right) w\right)+\left(c_{h},\left(\mathbf{u}_{h}-\mathbf{u}\right) \cdot \nabla w\right) .
$$

We denote by $q_{h}^{I}$ and $q_{h}^{P}$ the $L^{2}$-projections of $q^{I}$ and $q^{P}$ respectively, in the space $P_{h}$. We remark that $(2.9)$ yields

$$
\operatorname{div}\left(\mathbf{u}_{h}\right)=q_{h}^{I}-q_{h}^{P}
$$

Therefore we have

$$
\operatorname{div}\left(\mathbf{u}_{h}\right)-\operatorname{div}(\mathbf{u})=\left(q_{h}^{I}-q^{I}\right)-\left(q_{h}^{P}-q^{P}\right) .
$$


We can now obtain

$$
\lim _{h \rightarrow 0} \int_{0}^{T}\left(c_{h}, \operatorname{div}\left(\mathbf{u}_{h} w\right)\right)=\int_{0}^{T}(c, \operatorname{div}(\mathbf{u} w))
$$

From (3.31), we have

$$
\begin{gathered}
\lim _{h \rightarrow 0} \int_{0}^{T}\left(\mathbf{u}_{h} \cdot \nabla c_{h}, w_{h}\right)_{\mathcal{E}_{h}}=\lim _{h \rightarrow 0} \int_{0}^{T}\left(\mathbf{u}_{h} \cdot \nabla c_{h}, w\right)_{\mathcal{E}_{h}}, \\
\lim _{h \rightarrow 0} \int_{0}^{T}\left(w_{h}^{\text {down }} \mathbf{u}_{h} \cdot \mathbf{n}_{e},\left[c_{h}\right]\right)_{\Gamma_{h}}=\lim _{h \rightarrow 0} \int_{0}^{T}\left(w \mathbf{u}_{h} \cdot \mathbf{n}_{e},\left[c_{h}\right]\right)_{\Gamma_{h}} .
\end{gathered}
$$

Thus, from the result obtained in (3.50), (3.52)-(3.54) we have

$$
\begin{aligned}
\int_{0}^{T}(\mathbf{u} \cdot \nabla c, w) & =\int_{0}^{T}-(c, \operatorname{div}(\mathbf{u} w)) \\
& =\lim _{h \rightarrow 0} \int_{0}^{T}\left(\mathbf{u}_{h} \cdot \nabla c_{h}, w_{h}\right)_{\mathcal{E}_{h}}-\left(w_{h}^{\text {down }} \mathbf{u}_{h} \cdot \mathbf{n}_{e},\left[c_{h}\right]\right)_{\Gamma_{h}} .
\end{aligned}
$$

We have then proved (3.47) and we conclude that the limit $c$ satisfies the weak problem.

As a remark, further investigations are required for establishing the convergence of the solutions by NIPG and IIPG to the weak solution.

\section{Proof of COMPACTNESS TheOREM}

In this section, we prove Theorem 3.2. First, we fix $\delta>0$ and consider the space $L^{p}\left[\delta, T ; W\left(\mathcal{E}_{h}\right)\right]$. Its dual space is $L^{p^{\prime}}\left[\delta, T ; W\left(\mathcal{E}_{h}\right)^{\prime}\right]$ with $1 / p+1 / p^{\prime}=1$. Since the function $t \rightarrow w_{h}(t)-w_{h}(t-\delta)$ belongs to $W_{h}$, we use the definition of the projection $\Pi_{h}$ onto $W_{h}$ and its stability on $W\left(\mathcal{E}_{h}\right)$ to have:

$$
\begin{aligned}
\left(\int_{\delta}^{T}\left\|w_{h}(t)-w_{h}(t-\delta)\right\|_{W_{h}^{\prime}}^{p^{\prime}} \mathrm{d} t\right)^{1 / p^{\prime}} & =\sup _{v \in L^{p}\left[\delta, T ; W\left(\mathcal{E}_{h}\right)\right]} \frac{\int_{\delta}^{T}\left(w_{h}(t)-w_{h}(t-\delta), v\right)_{H} \mathrm{~d} t}{\|v\|_{L^{p}}\left[\delta, T ; W\left(\mathcal{E}_{h}\right)\right]} \\
& =\sup _{v \in L^{p}\left[\delta, T ; W\left(\mathcal{E}_{h}\right)\right]} \frac{\int_{\delta}^{T}\left(w_{h}(t)-w_{h}(t-\delta), \Pi_{h} v\right)_{H} \mathrm{~d} t}{\|v\|_{L^{p}\left[\delta, T ; W\left(\mathcal{E}_{h}\right)\right]}} \\
& =\sup _{v \in L^{p}\left[\delta, T ; W\left(\mathcal{E}_{h}\right)\right]} \frac{\int_{\delta}^{T}\left(w_{h}(t)-w_{h}(t-\delta), \Pi_{h} v\right)_{H} \mathrm{~d} t}{\left\|\Pi_{h} v\right\|_{L^{p}\left[\delta, T ; W\left(\mathcal{E}_{h}\right)\right]}} \frac{\left\|\Pi_{h} v\right\|_{L^{p}\left[\delta, T ; W\left(\mathcal{E}_{h}\right)\right]}}{\|v\|_{L^{p}\left[\delta, T ; W\left(\mathcal{E}_{h}\right)\right]}} \\
& \leq M \sup _{v \in L^{p}\left[\delta, T ; W\left(\mathcal{E}_{h}\right)\right]} \frac{\int_{\delta}^{T}\left(w_{h}(t)-w_{h}(t-\delta), \Pi_{h} v\right)_{H} \mathrm{~d} t}{\left\|\Pi_{h} v\right\|_{L^{p}\left[\delta, T ; W\left(\mathcal{E}_{h}\right)\right]}} .
\end{aligned}
$$

This implies

$$
\left(\int_{\delta}^{T}\left\|w_{h}(t)-w_{h}(t-\delta)\right\|_{W_{h}^{\prime}}^{p^{\prime}} \mathrm{d} t\right)^{1 / p^{\prime}} \leq M \sup _{v \in L^{p}\left[\delta, T ; W_{h}\right]} \frac{\int_{\delta}^{T}\left(w_{h}(t)-w_{h}(t-\delta), v\right)_{H} \mathrm{~d} t}{\|v\|_{L^{p}\left[\delta, T ; W\left(\mathcal{E}_{h}\right)\right]}} .
$$

Lemma 3.9 of [17] then gives that

$$
\sup _{v_{h} \in L^{p}\left[\delta, T ; W_{h}\right]} \frac{\int_{\delta}^{T}\left(w_{h}(t)-w_{h}(t-\delta), v_{h}\right)_{H} \mathrm{~d} t}{\left\|v_{h}\right\|_{L^{p}\left[\delta, T ; W\left(\mathcal{E}_{h}\right)\right]}} \leq M(\ell, \nu)\left\|F_{h}\right\|_{L^{q}\left[0, T ; W_{h}^{\prime}\right]} \max (\Delta t, \delta)^{1 / q^{\prime}} \delta^{1 / p^{\prime}} .
$$


Thus equation (4.1) becomes (with a different constant $M$ that depends on $\left\|\Pi_{h}\right\|_{\mathcal{L}\left(W\left(\mathcal{E}_{h}\right), W_{h}\right)}$ )

$$
\left(\int_{\delta}^{T}\left\|w_{h}(t)-w_{h}(t-\delta)\right\|_{W_{h}^{\prime}}^{p^{\prime}} \mathrm{d} t\right)^{1 / p^{\prime}} \leq M(\ell, \nu)\left\|F_{h}\right\|_{L^{q}\left[0, T ; W_{h}^{\prime}\right]} \max (\Delta t, \delta)^{1 / q^{\prime}} \delta^{1 / p^{\prime}} .
$$

Next, since $W \hookrightarrow W\left(\mathcal{E}_{h}\right)$, there is a constant $M>0$ such that

$$
\left\|w_{h}(t)-w_{h}(t-\delta)\right\|_{W^{\prime}} \leq M\left\|w_{h}(t)-w_{h}(t-\delta)\right\|_{W_{h}^{\prime}}
$$

Therefore we have

$$
\left(\int_{\delta}^{T}\left\|w_{h}(t)-w_{h}(t-\delta)\right\|_{W^{\prime}}^{p^{\prime}} \mathrm{d} t\right)^{1 / p^{\prime}} \leq M(\ell, \nu)\left\|F_{h}\right\|_{L^{q}\left[0, T ; W_{h}^{\prime}\right]} \max (\Delta t, \delta)^{1 / q^{\prime}} \delta^{1 / p^{\prime}} .
$$

By assumption, $\left\|F_{h}\right\|_{L^{q}\left[0, T ; W_{h}^{\prime}\right]}$ is uniformly bounded. We now show that $\left\{w_{h}\right\}_{h>0}$ is equicontinuous in $L^{p^{\prime}}\left[0, T ; W^{\prime}\right]$.

Fix $\epsilon>0$. We want to show there is $\delta_{0}>0$ such that

$$
\left(\int_{\delta}^{T}\left\|w_{h}(t)-w_{h}(t-\delta)\right\|_{W^{\prime}}^{p^{\prime}} \mathrm{d} t\right)^{1 / p^{\prime}} \leq \epsilon, \quad \forall h>0, \forall \delta<\delta_{0}
$$

Since $p>1$, we have $p^{\prime}<\infty$. If $q=1$, we choose $\delta_{0}$ such that $M \delta_{0}^{1 / p^{\prime}}<\epsilon$. If $q>1$, it suffices to find $\delta_{0}$ such that

$$
M \max \left(\Delta t, \delta_{0}\right)^{1 / q^{\prime}} \delta_{0}^{1 / p^{\prime}}<\epsilon .
$$

We can assume that $\delta_{0}<\Delta t$ and take

$$
\delta_{0}=\min \left(\frac{1}{2}\left(\frac{\epsilon}{M \Delta t^{1 / q^{\prime}}}\right)^{p^{\prime}}, \Delta t\right) .
$$

Consider now the case $q>1$, then $q^{\prime}<\infty$. It suffices to find $\delta_{0}$ such that

$$
M \max \left(\Delta t, \delta_{0}\right)^{1 / q^{\prime}} \delta_{0}^{1 / p^{\prime}}<\epsilon .
$$

We can assume that $\delta_{0}<\Delta t$ and choose

$$
\delta_{0}=\min \left(\frac{1}{2}\left(\frac{\epsilon}{M \Delta t^{1 / q^{\prime}}}\right)^{p^{\prime}}, \Delta t\right)
$$

By assumption $\left\{w_{h}\right\}_{h>0}$ is bounded in $L^{p}[0, T ; V]$ with $p>1$. This implies that $\left\{w_{h}\right\}_{h>0}$ is bounded in $L^{1}[0, T ; V]$. In addition, we showed that $\left\{w_{h}\right\}_{h>0}$ is equicontinuous in $L^{p^{\prime}}\left[0, T ; W^{\prime}\right]$ for $1<p^{\prime}<\infty$. Then, from Theorem 3.2 of [21], we conclude that for all $0<\theta<T / 2$, the set $\left\{\left.w_{h}\right|_{(\theta, T-\theta)}\right\}_{h>0}$ is precompact in $L^{p^{\prime}}\left[\theta, T-\theta ; W^{\prime}\right]$.

Equation (4.2), with the assumption $0<\delta<T$, gives:

$$
\int_{\delta}^{T}\left\|w_{h}(t)-w_{h}(t-\delta)\right\|_{W^{\prime}}^{p^{\prime}} \mathrm{d} t \leq M \delta
$$

Using Lemma 3.4 in [21], we conclude that $\left\{w_{h}\right\}_{h>0}$ is uniformly bounded in $L^{r}\left[0, T ; W^{\prime}\right]$ for any $1 \leq r<\infty$. Therefore uniform integrability holds and this implies that $\left\{w_{h}\right\}_{h>0}$ is precompact in $L^{p^{\prime}}\left[0, T ; W^{\prime}\right]$. Now, the fact 
that $\left\{w_{h}\right\}_{h>0}$ is bounded in $L^{r}\left[0, T ; W^{\prime}\right]$ for any $1 \leq r<\infty$ and that $\left\{w_{h}\right\}_{h>0}$ is precompact in $L^{p^{\prime}}\left[0, T ; W^{\prime}\right]$, implies that $\left\{w_{h}\right\}_{h>0}$ is precompact in $L^{r}\left[0, T ; W^{\prime}\right]$ for any $1 \leq r<\infty$.

Finally it remains to show that $\left\{w_{h}\right\}_{h>0}$ is precompact in $L^{p}[0, T ; H]$. From [19] the fact $V \hookrightarrow H \hookrightarrow W^{\prime}$ implies that for all $\epsilon>0$ there exists $M(\epsilon)>0$ such that

$$
\left\|w_{h}(t)\right\|_{H} \leq \epsilon\left\|w_{h}(t)\right\|_{V}+M(\epsilon)\left\|w_{h}(t)\right\|_{W^{\prime}} .
$$

So,

$$
\left\|w_{h}\right\|_{L^{p}[0, T ; H]} \leq \epsilon\left\|w_{h}\right\|_{L^{p}[0, T ; V]}+M(\epsilon)\left\|w_{h}\right\|_{L^{p}\left[0, T ; W^{\prime}\right]} .
$$

Since $\left\{w_{h}\right\}_{h>0}$ is bounded in $L^{p}[0, T ; V]$ and precompact in $L^{p}\left[0, T ; W^{\prime}\right]$ it easily follows that it is also precompact in $L^{p}[0, T ; H]$.

\section{NumericAl EXAMPLE}

We test our method for the following analytical solutions on the unit square:

$$
\begin{aligned}
& p(x, y, t)=\left(2-\mathrm{e}^{-x}\left(1+x+x^{2}\right)-\mathrm{e}^{-y}\left(1+y+y^{2}\right)\right) \mathrm{e}^{\frac{\pi t}{2}}, \\
& c(x, y, t)=\frac{1}{2}\left(\sin (2 \pi x)^{2}+\cos (2 \pi y)^{2}\right) \sin \left(\frac{\pi t}{2}\right) .
\end{aligned}
$$

The parameters in the equations are

$$
\begin{aligned}
& \phi=0.2, \mathbf{K}(c)=\frac{9.44 \times 10^{-3}}{1+(0.0524 c)^{4.74}}, \mathbf{g}=\mathbf{0}, q^{I}=1, \epsilon=\sigma=1, \\
& \mathbf{D}(\mathbf{u})=\frac{\mathbf{u u}^{T}}{|\mathbf{u}|}\left(\alpha_{\ell}-\alpha_{t}\right)+\left(d_{m}+\alpha_{t}|\mathbf{u}|\right) \mathbf{I}, \quad \alpha_{\ell}=1.8 \times 10^{-5}, \alpha_{t}=0.1 \alpha_{\ell}, d_{m}=1.8 \times 10^{-7} .
\end{aligned}
$$

A fourth order method in time is used. We vary the degree of approximation in space from a first order method to a third order method. In order to reduce the size of the systems, we decouple the velocity-pressure equations from the concentration equation. Table 1 shows the errors and convergence rates for the first-order method in space $(k=0$ and $r=1)$ obtained at the final time $t=0.5$. We then increase the order of the method and shows the corresponding results in Tables $2-3$. For all simulations, the time step is chosen constant equal to $10^{-2}$. The numerical rates show convergence of the proposed method.

\section{Appendix A.}

Lemma A.1. Let $E$ be a mesh element and let e be a face of $\partial E$. For any polynomial function $w_{h}$ defined on $E$, and any polynomial vector function $\mathbf{z}_{h}$ defined on $E$, we have

$$
\begin{aligned}
\left\|\mathbf{D}^{1 / 2}\left(\mathbf{z}_{h}\right) \nabla w_{h}\right\|_{L^{2}(e)} & \lesssim h^{-1 / 2}\left(\left\|\nabla w_{h}\right\|_{L^{2}(E)}^{2}+\left\|\left|\mathbf{z}_{h}\right|^{1 / 2}\left|\nabla w_{h}\right|\right\|_{L^{2}(E)}^{2}\right)^{1 / 2} \\
\left\|\mathbf{D}^{1 / 2}\left(\mathbf{z}_{h}\right) \nabla w_{h}\right\|_{L^{2}(e)} & \lesssim h^{-1 / 2}\left(\left\|\nabla w_{h}\right\|_{L^{2}(E)}+\left\|\mathbf{z}_{h}\right\|_{L^{2}(E)}^{1 / 2}\left\|\nabla w_{h}\right\|_{L^{4}(E)}\right)
\end{aligned}
$$

Proof. With (2.7), we have

$$
\left\|\mathbf{D}^{1 / 2}\left(\mathbf{z}_{h}\right) \nabla w_{h}\right\|_{L^{2}(e)} \lesssim\left(\int_{e}\left(1+\left|\mathbf{z}_{h}\right|\right)\left|\nabla w_{h}\right|^{2}\right)^{1 / 2} \lesssim\left(\left\|\nabla w_{h}\right\|_{L^{2}(e)}^{2}+\left\|\left|\mathbf{z}_{h}\right|^{1 / 2}\left|\nabla w_{h}\right|\right\|_{L^{2}(e)}^{2}\right)^{1 / 2} .
$$

We now make use of a standard trace inequality for polynomial functions

$$
\left\|\nabla w_{h}\right\|_{L^{2}(e)} \lesssim h^{-1 / 2}\left\|\nabla w_{h}\right\|_{L^{2}(E)} .
$$


TABLE 1. Errors and rates for method of first-order in space.

\begin{tabular}{ccccc}
\hline \multicolumn{5}{c}{ Pressure and velocity } \\
\hline$h$ & $\left\|p-p_{h}\right\|_{L^{2}(\Omega)}$ & Cvg. rate & $\left\|\mathbf{u}-\mathbf{u}_{h}\right\|_{L^{2}\left(\mathcal{E}_{h}\right)}$ & Cvg. rate \\
\hline $2^{-2}$ & $5.556826 \mathrm{e}-2$ & - & $2.651639 \mathrm{e}-4$ & - \\
$2^{-3}$ & $1.940921 \mathrm{e}-2$ & 1.52 & $6.730343 \mathrm{e}-5$ & 1.98 \\
$2^{-4}$ & $7.540286 \mathrm{e}-3$ & 1.36 & $1.689074 \mathrm{e}-5$ & 1.99 \\
$2^{-5}$ & $3.371601 \mathrm{e}-3$ & 1.16 & $4.226787 \mathrm{e}-6$ & 2.00 \\
$2^{-6}$ & $1.626564 \mathrm{e}-3$ & 1.05 & $1.056954 \mathrm{e}-6$ & 2.00 \\
$2^{-7}$ & $8.053275 \mathrm{e}-4$ & 1.01 & $2.642547 \mathrm{e}-7$ & 2.00 \\
\hline \multicolumn{5}{c}{ Concentration } \\
\hline$h$ & $\left\|c-c_{h}\right\|_{L^{2}(\Omega)}$ & Cvg. rate & $\left\|\nabla c-\nabla c_{h}\right\|_{L^{2}\left(\mathcal{E}_{h}\right)}$ & Cvg. rate \\
\hline $2^{-2}$ & $2.126244 \mathrm{e}-2$ & - & $4.860163 \mathrm{e}-1$ & - \\
$2^{-3}$ & $1.572547 \mathrm{e}-2$ & 0.44 & $9.787947 \mathrm{e}-1$ & -1.01 \\
$2^{-4}$ & $4.070491 \mathrm{e}-3$ & 1.95 & $5.012288 \mathrm{e}-1$ & 0.97 \\
$2^{-5}$ & $1.050597 \mathrm{e}-3$ & 1.95 & $2.532644 \mathrm{e}-1$ & 0.98 \\
$2^{-6}$ & $2.790473 \mathrm{e}-4$ & 1.91 & $1.284574 \mathrm{e}-1$ & 0.98 \\
$2^{-7}$ & $7.677982 \mathrm{e}-5$ & 1.86 & $6.579732 \mathrm{e}-2$ & 0.97 \\
\hline
\end{tabular}

TABLE 2. Errors and rates for method of second order in space.

\begin{tabular}{ccccc}
\hline \multicolumn{5}{c}{ Pressure and velocity } \\
\hline$h$ & $\left\|p-p_{h}\right\|_{L^{2}(\Omega)}$ & Cvg. rate & $\left\|\mathbf{u}-\mathbf{u}_{h}\right\|_{L^{2}\left(\mathcal{E}_{h}\right)}$ & Cvg. rate \\
\hline $2^{-1}$ & $3.75612627 \mathrm{e}-2$ & - & $9.12292627 \mathrm{e}-5$ & - \\
$2^{-2}$ & $1.51988594 \mathrm{e}-2$ & 1.305 & $1.18909883 \mathrm{e}-2$ & 2.940 \\
$2^{-3}$ & $4.70278178 \mathrm{e}-3$ & 1.692 & $1.50219946 \mathrm{e}-6$ & 2.985 \\
$2^{-4}$ & $1.30205703 \mathrm{e}-3$ & 1.853 & $1.88274337 \mathrm{e}-7$ & 2.996 \\
$2^{-5}$ & $3.42238663 \mathrm{e}-4$ & 1.928 & $2.35499363 \mathrm{e}-8$ & 2.999 \\
\hline \multicolumn{5}{c}{ Concentration } \\
\hline$h$ & $\left\|c-c_{h}\right\|_{L^{2}(\Omega)}$ & Cvg. rate & $\left\|\nabla c-\nabla c_{h}\right\|_{L^{2}\left(\mathcal{E}_{h}\right)}$ & Cvg. rate \\
\hline $2^{-1}$ & $2.32838162 \mathrm{e}-3$ & - & 1.86209926 & - \\
$2^{-2}$ & $1.37922246 \mathrm{e}-3$ & 0.755 & 1.10375937 & 0.755 \\
$2^{-3}$ & $2.30891708 \mathrm{e}-4$ & 2.579 & $2.16460653 \mathrm{e}-1$ & 2.350 \\
$2^{-4}$ & $4.84970125 \mathrm{e}-5$ & 2.251 & $5.47554909 \mathrm{e}-2$ & 1.983 \\
$2^{-5}$ & $1.01955089 \mathrm{e}-5$ & 2.250 & $1.36293565 \mathrm{e}-2$ & 2.006 \\
\hline
\end{tabular}

Let $z_{h, i}$ denote the $i$ th component of $\mathbf{z}_{h}$.

$$
\begin{aligned}
\left\|\left|\mathbf{z}_{h}\right|^{1 / 2}\left|\nabla w_{h}\right|\right\|_{L^{2}(e)} & \lesssim|e|^{1 / 4}\left(\int_{e}\left|\mathbf{z}_{h}\right|^{2}\left|\nabla w_{h}\right|^{4}\right)^{1 / 4} \lesssim|e|^{1 / 4}\left(\sum_{i, j=1}^{d} \int_{e} z_{h, i}^{2}\left(\frac{\partial w_{h}}{\partial x_{j}}\right)^{4}\right)^{1 / 4} \\
& \lesssim|e|^{1 / 4}\left(\sum_{i, j=1}^{d}\left\|z_{h, i}\left(\frac{\partial w_{h}}{\partial x_{j}}\right)^{2}\right\|_{L^{2}(e)}^{2}\right)^{1 / 4} \\
& \lesssim|e|^{1 / 4}\left(h^{-1} \sum_{i, j=1}^{d}\left\|z_{h, i}\left(\frac{\partial w_{h}}{\partial x_{j}}\right)^{2}\right\|_{L^{2}(E)}^{2}\right)^{1 / 4}
\end{aligned}
$$


TABLE 3. Errors and rates for method of third order in space.

\begin{tabular}{ccccc}
\hline \multicolumn{5}{c}{ Pressure and Velocity } \\
\hline$h$ & $\left\|p-p_{h}\right\|_{L^{2}(\Omega)}$ & Cvg. rate & $\left\|\mathbf{u}-\mathbf{u}_{h}\right\|_{L^{2}\left(\mathcal{E}_{h}\right)}$ & Cvg. rate \\
\hline $2^{-1}$ & $1.14265447 \mathrm{e}-2$ & - & $4.08259591 \mathrm{e}-6$ & - \\
$2^{-2}$ & $1.81358816 \mathrm{e}-3$ & 2.655 & $2.62942571 \mathrm{e}-7$ & 3.957 \\
$2^{-3}$ & $2.55346692 \mathrm{e}-4$ & 2.828 & $1.65601216 \mathrm{e}-8$ & 3.989 \\
$2^{-4}$ & $3.38735338 \mathrm{e}-5$ & 2.914 & $1.03699484 \mathrm{e}-9$ & 3.997 \\
$2^{-5}$ & $4.36191826 \mathrm{e}-6$ & 2.957 & $6.48427172 \mathrm{e}-11$ & 3.999 \\
\hline \multicolumn{5}{c}{ Concentration } \\
\hline$h$ & $\left\|c-c_{h}\right\|_{L^{2}(\Omega)}$ & Cvg. rate & $\left\|\nabla c-\nabla c_{h}\right\|_{L^{2}\left(\mathcal{E}_{h}\right)}$ & Cvg. rate \\
\hline $2^{-1}$ & $2.19480438 \mathrm{e}-3$ & - & 1.78168909 & - \\
$2^{-2}$ & $1.00751042 \mathrm{e}-4$ & 4.445 & $8.09128327 \mathrm{e}-2$ & 4.461 \\
$2^{-3}$ & $3.20635143 \mathrm{e}-5$ & 1.652 & $3.15839214 \mathrm{e}-2$ & 1.357 \\
$2^{-4}$ & $3.69915500 \mathrm{e}-6$ & 3.116 & $4.05515779 \mathrm{e}-3$ & 2.961 \\
$2^{-5}$ & $4.80717242 \mathrm{e}-7$ & 2.944 & $5.14981839 \mathrm{e}-4$ & 2.977 \\
\hline
\end{tabular}

By an inverse inequality, we have

$$
\left\|z_{h, i}\left(\frac{\partial w_{h}}{\partial x_{j}}\right)^{2}\right\|_{L^{2}(E)} \lesssim h^{-d / 2}\left\|z_{h, i}\left(\frac{\partial w_{h}}{\partial x_{j}}\right)^{2}\right\|_{L^{1}(E)}
$$

Therefore, we can conclude

$$
\begin{aligned}
\left\|\left|\mathbf{z}_{h}\right|^{1 / 2}\left|\nabla w_{h}\right|\right\|_{L^{2}(e)} & \lesssim|e|^{1 / 4}\left(h^{-1} h^{-d} \sum_{i, j=1}^{d}\left\|z_{h, i}\left(\frac{\partial w_{h}}{\partial x_{j}}\right)^{2}\right\|_{L^{1}(E)}^{2}\right)^{1 / 4} \\
& \lesssim h^{-1 / 2}\left(\sum_{i, j=1}^{d}\left(\int_{E}\left|z_{h, i}\right|\left(\frac{\partial w_{h}}{\partial x_{j}}\right)^{2}\right)^{2}\right)^{1 / 4} \\
& \lesssim h^{-1 / 2}\left\|\left|\mathbf{z}_{h}\right|^{1 / 2}\left|\nabla w_{h}\right|\right\|_{L^{2}(E)} .
\end{aligned}
$$

Combining (A.3) and (A.4) yields (A.1). To obtain (A.2), we apply Cauchy-Schwarz's inequality to (A.1).

$$
\left\|\mathbf{D}^{1 / 2}\left(\mathbf{z}_{h}\right) \nabla w_{h}\right\|_{L^{2}(e)} \lesssim h^{-1 / 2}\left(\left\|\nabla w_{h}\right\|_{L^{2}(E)}^{2}+\left\|\mathbf{z}_{h}\right\|_{L^{2}(E)}\left\|\nabla w_{h}\right\|_{L^{4}(E)}^{2}\right)^{1 / 2} .
$$

\section{REFERENCES}

[1] S. Bartels, M. Jensen and R. Müller, Discontinuous Galerkin finite element convergence for incompressible miscible displacement problems of low regularity. SIAM J. Numer. Anal. 47 (2009) 3720-3743.

[2] S. Brenner and R. Scott, The Mathematical Theory of Finite Element Methods. In vol. 15. Springer-Verlag (2007).

[3] F. Brezzi, J. Douglas, M. Fortin and L. Marini, Efficient rectangular mixed finite elements in two and three space variables. RAIRO Model. Math. Anal. Numer. 21 (1987) 581-604.

[4] F. Brezzi and M. Fortin, Mixed and hybrid finite element methods. Number 15 in Comput. Math. Springer-Verlag (1991).

[5] A. Buffa and C. Ortner, Variational convergence of IP-DGFEM. Technical Report (2007).

[6] A. Buffa and C. Ortner, Compact embeddings of broken Sobolev spaces and applications. IMA J. Numer. Anal. 29 (2009) $827-855$.

[7] Z. Chen and R.E. Ewing, Mathematical analysis for reservoir models. SIAM J. Math. Anal. 30 (1999) $431-453$.

[8] D. Di Pietro and A. Ern, Discrete functional analysis tools for discontinuous Galerkin methods with application to the incompressible Navier-Stokes equations. Math. Comput. 79 (2010) 1303-1330. 
[9] J. Douglas, R.E. Ewing and M.F. Wheeler, A time-discretization procedure for a mixed finite element approximation of miscible displacement in porous media. RAIRO. Numer. Anal. 17 (1983) 249-265.

[10] Y. Epshteyn and B.B. Rivière, Convergence of high order methods for miscible displacement. Int. J. Numer. Anal. Model. 5 (2008) 47-63.

[11] K. Eriksson, C. Johnson and V. Thomée, Time discretization of parabolic problems by the discontinuous Galerkin method. RAIRO Model. Math. Anal. Numer. 19 (1985) 611-643.

[12] R. Ewing and M.F. Wheeler, Galerkin methods for miscible displacement problems in porous media. SIAM J. Numer. Anal. 17 (1980) 351-365.

[13] R.E. Ewing and T. Russell, Efficient time-stepping methods for miscible displacement problems in porous media. SIAM J. Numer. Anal. 19 (1982) 1-67.

[14] X. Feng, On existence and uniqueness results for a coupled system modeling miscible displacement in porous media. J. Math. Anal. Appl. 194 (1995) 883-910.

[15] M. Jensen and R. Müller, Stable Crank-Nicolson discretisation for incompressible miscible displacement problems of low regularity. Numer. Math. Adv. Appl. (2010) 469-477.

[16] M. Ohlberger, Convergence of a mixed finite element - finite volume method for the two phase flow in porous media. East-Weat J. Numer. Math. 5 (1997) 183-210.

[17] B. Riviere and N.J. Walkington, Convergence of a discontinuous Galerkin method for the miscible displacement under low regularity. SIAM J. Numer. Anal. 49 (2011) 1085-1110.

[18] T.F. Russell, Time stepping along characteristics with incomplete iteration for a Galerkin approximation of miscible displacement in porous media. SIAM J. Numer. Anal. 22 (1985) 970-1013.

[19] R.E. Showalter, Monotone operators in Banach space and nonlinear partial differential equations. American Mathematical Society, Providence, RI (1997). Available online at http://www.ams.org/online_bks/surv49/.

[20] S. Sun, B. Riviere and M.F. Wheeler, A combined mixed finite element and discontinuous Galerkin method for miscible displacement problem in porous media. Recent Progress in Computational and Applied PDEs (2002) 323-348.

[21] N.J. Walkington, Compactness properties of the DG and CG time stepping schemes for parabolic equations. SIAM J. Numer. Anal. 47 (2010) 4680-4170. 